\title{
Nascent alt-protein chemoproteomics reveals a repressor of ribosome biogenesis
}

\section{Sarah Slavoff ( $\nabla$ sarah.slavoff@yale.edu )}

Yale University https://orcid.org/0000-0002-4443-2070

\section{Xiongwen Cao}

Yale University

Alexandra Khitun

Yale University

Cecelia Harold

Yale University

\section{Carson Bryant}

Yale University https://orcid.org/0000-0002-7475-875X

Shu-Jian Zheng

Yale University

\section{Susan Baserga}

Yale University School of Medicine

\section{Article}

Keywords: alt-proteins, MINAS-60, ribosome biogenesis, chemoproteomics

Posted Date: September 9th, 2021

DOI: https://doi.org/10.21203/rs.3.rs-871945/v1

License: (c) (i) This work is licensed under a Creative Commons Attribution 4.0 International License.

Read Full License 
2 Nascent alt-protein chemoproteomics reveals a repressor of ribosome 3 biogenesis

4

5 Xiongwen $\mathrm{Cao}^{1,2}$, Alexandra Khitun ${ }^{1,2}$, Cecelia M. Harold ${ }^{3}$, Carson J. Bryant ${ }^{4}$,

6 Shu-Jian Zheng ${ }^{1,2}$, Susan J. Baserga ${ }^{3,4,5}$, Sarah A. Slavoff ${ }^{1,2,4}$

$8{ }^{1}$ Department of Chemistry, Yale University, New Haven, Connecticut 06520,

$9 \quad$ United States

102 Institute of Biomolecular Design and Discovery, Yale University, West Haven,

11 Connecticut 06516, United States

12 3Department of Genetics, Yale University School of Medicine, New Haven,

13 Connecticut 06520, United States

14 4Department of Molecular Biophysics and Biochemistry, Yale University, New

15 Haven, Connecticut 06520, United States

$16{ }^{5}$ Department of Therapeutic Radiology, Yale University School of Medicine, New

17 Haven, Connecticut 06520, United States

18 *Correspondence: sarah.slavoff@yale.edu

\section{Abstract}

21 Many unannotated microproteins and alternative proteins (alt-proteins) have

22 recently been found to be co-encoded with canonical proteins, but few of their

23 functions are known. Motivated by the hypothesis that alt-proteins undergoing

24 active or stress-induced synthesis could play important cellular roles, here, we

25 developed a chemoproteomic pipeline to identify nascent alt-proteins in human

26 cells. We identified 22 actively translated unannotated alt-proteins, one of which

27 is upregulated after DNA damage stress. We further defined MINAS-60

28 (MIcroprotein that Negatively regulates ASsembly of the pre-60S ribosomal

29 subunit), a nucleolar localized alt-protein co-encoded with human RBM10. 
Depletion of MINAS-60 increases the amount of the mature 605 ribosomal subunit, consequently upregulating global protein synthesis and cell proliferation by repressing late-stage pre-60S assembly and export of the 60S ribosome subunit to the cytoplasm. Together, these results implicate MINAS-60 as a repressor of ribosome biogenesis, and demonstrate that chemoproteomics can enable generation of functional hypotheses for uncharacterized alt-proteins.

\section{Introduction}

Expression of thousands of previously unannotated small open reading frames (smORFs, typically defined as ORFs comprising fewer than 100 codons and, in some studies, up to 150 codons $^{1}$ ) has recently been revealed in mammalian cells ${ }^{2}$. These smORFs are found in long non-coding RNAs, 5' and $3^{\prime}$ untranslated regions (UTRs) of mRNAs, and frame-shifted ORFs overlapping protein coding sequences (CDS), the latter of which are termed alternative ORFs or "alt-ORFs"3. A rapidly increasing number of smORF-encoded proteins (SEPs, also known as micropeptides and microproteins) and alt-ORF-encoded proteins (alt-proteins) ${ }^{2}$ have been shown to play important roles in vertebrate biology. For example, the human SEPs BRAWNIN and MOCCI function in oxidative phosphorylation ${ }^{4,5}$, and MP31 is a tumor suppressor that regulates lactate metabolism in glioblastoma ${ }^{6}$. Fewer alt-proteins have been well-defined, including alt-FUS, which cooperates with FUS in formation of cytotoxic aggregates ${ }^{7}$, and alt-RPL36, which regulates the PI3K-AKT-mTOR pathway ${ }^{8}$. Recently, genome-scale CRISPR screens revealed that hundreds of smORFs regulate human cell growth and survival ${ }^{9,10}$. These studies demonstrate that defining the function of bioactive SEPs and altproteins represents a major opportunity to gain insights into biological complexity.

Currently, there is proteomic and ribosome profiling evidence for tens of thousands of human smORFs and alt-ORFs ${ }^{3,11}$, but the overwhelming majority of smORFs and alt-ORFs remain uncharacterized, in part because their short lengths and, in some cases, limited conservation render homology-based annotation challenging ${ }^{12}$. Furthermore, most alt-ORFs remain unstudied because 
61 it is challenging to separate their functions from the canonical protein CDS in

62 which they are nested. Because strategies to identify alt-proteins that participate

63 in biological processes are currently in the developmental stages, it remains

64 unclear whether alt-proteins are broadly functional. We hypothesize that alt-

65 proteins with properties (e.g., chemical reactivity, regulated expression) similar to

66 those of canonical proteins are likely to play important cellular roles, and that

67 chemoproteomic strategies targeted to those properties can be leveraged to

68 identify functional alt-proteins. Providing precedent, a chemoproteomic profiling study of microproteins containing reactive cysteine residues, which is a feature of the active site of many enzymes, revealed 16 nucleophilic microproteins, though

71 their cellular roles were not explored in that study ${ }^{13}$. In this work, we test the

72 hypothesis that alt-proteins undergoing active or stress-induced synthesis are

73 likely to be functional, and we develop a chemoproteomic approach to identify 74 them.

Using this method, we identify an alt-ORF that overlaps the human RBM10 CDS and encodes a repressor of ribosome large subunit (LSU) biogenesis. Ribosome

78 biogenesis is a highly spatially and temporally regulated cellular process essential for growth and development ${ }^{14-17}$. In humans, ribosome biogenesis starts in the nucleolus with the transcription of a $47 \mathrm{~S}$ precursor rRNA (pre-rRNA)

81 by RNA polymerase I (RNAPI). The $47 \mathrm{~S}$ pre-rRNA is chemically modified and complexed with ribosome assembly factors and ribosomal proteins to form the $90 \mathrm{~S}$ pre-ribosomal particle. Endonucleolytic cleavage of the $47 \mathrm{~S}$ pre-rRNA subsequently generates the pre-40S and pre-60S particles, which undergo individual maturation and quality-control steps prior to export to the cytoplasm.

86 Pre-60S ribosomal particles have recently been probed by cryo-electron 87 microscopy (cryo-EM) ${ }^{18,19}$, revealing a sequential maturation process involving 88 quality control checkpoints for $5 \mathrm{~S}$ ribonucleoprotein particle incorporation and rotation ${ }^{20}$, active site formation, and removal of internal transcribed spacer 2

90 (ITS2) prior to export from the nucleus to the cytoplasm. In the cytoplasm, the

91 final steps of maturation occur to produce large $60 \mathrm{~S}$ and small $40 \mathrm{~S}$ subunits, 
92 which associate to form translation-competent ribosomes. Dysregulated

93 ribosome biogenesis has been linked to numerous human disorders, including

94 cancer $^{21}$, Alzheimer's disease ${ }^{22}$, and congenital disorders termed

95 ribosomopathies ${ }^{23,24}$.

96

97

\section{Results}

\section{Chemoproteomic profiling of nascent alt-proteins}

117 Motivated by the hypothesis that alt-proteins undergoing active synthesis or 118 stress-induced synthesis could play important cellular roles, we set out to 119

In this study, we developed a chemoproteomic pipeline to identify nascent altproteins, which modifies a powerful previously reported strategy for metabolic unnatural amino acid incorporation to be amenable to microprotein enrichment and profiling, and identified 22 unannotated alt-proteins in a human cell line. We confirmed the translation of six selected alt-proteins, and functionally defined one alt-ORF nested in the human RBM10 CDS. We name the encoded alt-protein MINAS-60, or MIcroprotein that Negatively regulates ASsembly of the pre-60S ribosomal subunit. We show that MINAS-60 localizes to the nucleolus, associates with LSU assembly factors GTPBP4 and MRTO4, and co-fractionates with pre-60S complexes in nuclear extracts. Finally, we engineer MINAS-60 knockdown and rescue cell lines to demonstrate that loss of MINAS-60 increases cytoplasmic $60 \mathrm{~S}$ ribosome subunit levels, global protein synthesis, and cell proliferation. This is independent of the function of the canonical RBM10 protein, which has been previously shown to inhibit cell proliferation through its role in alternative pre-mRNA splicing ${ }^{25-29}$. These results implicate MINAS-60 as a rare negative regulator of late nuclear steps in LSU biogenesis, and demonstrate that chemoproteomic profiling can prioritize alt-ORFs for functional study. develop a chemoproteomic approach to identify newly translated alt-proteins (Figure 1a). We leveraged bio-orthogonal non-canonical amino acid tagging (BONCAT), an in vivo labeling strategy to identify nascent proteins ${ }^{30}$. BONCAT utilizes the methionine analogue azidohomoalanine (AHA), which can be 
123 metabolically incorporated into all newly synthesized proteins by the endogenous

124 protein translation machinery. Previous BONCAT workflows included procedures, 125 such as column-based biotin removal, that were likely to eliminate small proteins.

126 We modified the protocol to capture these small proteins, including microproteins

127 and alt-proteins. Labeled (and unlabeled) small proteins were selectively

128 enriched from whole proteome extracts with a C8 column following a previously

129 reported strategy ${ }^{31}$ (Figure $1 \mathrm{~b}$ ), and AHA-containing small proteins were captured 130 with click chemistry directly on dibenzocyclooctyne (DBCO) magnetic beads. On-

131 bead digest was followed with our previously reported liquid

132 chromatography/tandem mass spectrometry-based method for identification of

133 unannotated microproteins and alt-proteins ${ }^{32}$. Using this strategy, we profiled

134 translation in HEK 293T cells under basal growth conditions (DMSO treatment),

135 oxidative stress (sodium arsenite treatment), DNA damage stress (etoposide

136 treatment) and unfolded protein response stress (DTT treatment). In total, we

137 identified 22 unannotated alt-proteins (Figure 1c, Supplementary Data 1), nine of

138 which were specifically detected under stress conditions, including alt-CNPY2,

139 which was specifically detected after etoposide treatment (Figure 1d,

140 Supplementary Data 1).

141

142 To confirm the translation and examine subcellular localization of six selected alt-

143 proteins (Supplementary Figures 1a-f, Supplementary Table 1), the cDNA

144 sequence comprising the 5'UTR of the encoding transcript through the stop

145 codon of the putative alt-ORF was cloned into a mammalian expression vector

146 with a FLAG-HA epitope tag on the C-terminus of the alt-protein, followed by

147 transfection and immunostaining. As shown in Figure 1e, over-expressed alt-

148 DRAP1 (previously validated ${ }^{12}$ and included as a positive control), alt-PRR3, alt-

149 PRH1, alt-CNPY2 and alt-CACTIN were nucleocytoplasmic. Over-expressed

150 MINAS-60 co-localized with a nucleolar protein, fibrillarin (Figure 1f). Western

151 blotting further confirmed the translation of the six alt-proteins (Figure 1g).

152 MINAS-60 and alt-CNPY2 produced two immunoreactive bands, which is due to

153 multiple in-frame start codon for MINAS-60 (vide infra), and may be caused by 
154 multiple start sites, phosphorylation or other post-translational modification for alt-

155 CNPY2, analogous to alt-RPL36 ${ }^{8}$.

We hypothesized that the alt-proteins detected specifically under stress conditions are induced in response to cellular stress. To determine whether the expression of alt-CNPY2 is induced by DNA damage, we treated HEK 293T over-expressing alt-CNPY2 with etoposide, followed by western blotting. As shown in Figure $1 \mathrm{~h}$, alt-CNPY2 is upregulated up to two fold upon etoposide treatment in both dose- and time-dependent manner. However, the mRNA level of CNPY2 did not change (Supplementary Figure 1g), indicating that upregulation of alt-CNPY2 is likely due to increased translation or decreased proteolysis, but not increased transcription. Taken together, these results suggest that our chemoproteomic pipeline is able to detect nascent or stress-induced alt-proteins.

\section{MINAS-60 is cell-cycle regulated and conserved in mouse}

We selected MINAS-60 from the BONCAT-detected microprotein dataset for further study because it localizes to the nucleolus, and only one nucleolar microprotein has previously been identified ${ }^{33}$; furthermore, MINAS-60 is nested within the RBM10 CDS, and therefore probing its cellular and molecular roles could shed light on the poorly characterized class of overlapping alt-proteins (Figure 2a, Supplementary Data 2). A different MINAS-60 tryptic peptide was previously detected in human colorectal cancer samples, supporting expression of MINAS-60 in human tissue, but the alt-protein was not defined or characterized in that study ${ }^{34}$. To validate expression of endogenous MINAS-60 from the RBM10 genomic locus, we generated two independent Cas9-directed knock-in (KI) HEK 293T cell lines with a 3×GFP11-FLAG-HA tag appended to the 3 ' end of MINAS-60 alt-ORF ${ }^{35}$, followed with immunostaining. As shown in Figure $2 b$, endogenously expressed MINAS-60 co-localizes with fibrillarin, consistent with the over-expression results, suggesting MINAS-60 likely functions in the nucleolus. 
The nucleolus is the site of ribosome biogenesis in eukaryotic cells. Ribosome biogenesis starts with the transcription of rDNA, which oscillates during the cell cycle, nearly ceasing during $M$ phase, increasing during $G 1$ phase, and maximizing during $S$ and $G 2$ phases in human cells ${ }^{36-39}$. If MINAS-60 regulates ribosome biogenesis, we hypothesized that the expression of nucleolar MINAS60 would be correlated with ribosome biogenesis during the cell cycle. Immunostaining of synchronized MINAS-60 KI HEK 293T cells revealed that nucleolar MINAS-60 staining intensity increased at early S phase, peaking at the $2 \mathrm{~h}$ time point. MINAS-60 expression then decreased by late $S$ phase, and was very low during G2/M phase. At G1 phase, the MINAS-60 staining intensity again increased (Supplementary Figure 2). These results were confirmed with western blotting (Figure 2c). MINAS-60 expression is therefore coordinated with ribosome biogenesis activity during the cell cycle.

We then identified the start codon(s) that initiate MINAS-60 translation. Alt-ORFs have been reported to initiate at upstream, non-AUG start codons $8,12,40$ and internal, AUG start codons ${ }^{7,41}$. We tested two upstream in-frame non-AUG start codons, $\mathrm{A}_{383} \mathrm{TC}$ and $\mathrm{A}_{386} \mathrm{GG}$ (numbered relative to the first nucleotide of the cDNA), as well as seven internal AUG start codons (numbered ATG1 - ATG7) (Supplementary Figure 3a). The $\sim 20 \mathrm{kDa}$ MINAS-60 isoform likely initiates at ATG1 and the $\sim 15 \mathrm{kDa}$ MINAS-60 isoform likely initiates at ATG6 or 7, because the indicated species is abrogated only when the corresponding start codon is deleted or mutated (Supplementary Figures b-c). These results were further confirmed by over-expressing truncated MINAS-60 coding sequences starting from ATG1 or ATG6 and comparing their products' sizes with the wild-type construct (Supplementary Figure 3d). The MINAS-60 smORF is therefore entirely contained within the RBM10 coding sequence, and the MINAS-60 ATG1 start codon is only 7 nucleotides downstream of the RBM10 start codon (Figure 2a). We therefore speculate that MINAS-60 translation may initiate within RBM10 transcript variant 1 via leaky scanning, and that the additional MINAS-60 isoform 
215 diversity observed in $\mathrm{KI}$ cells could be generated by alternative splicing,

216 analogous to PTBP3 ${ }^{41}$.

ClustalW alignment of hypothetical MINAS-60 homologs from mouse, cattle, and monkey revealed significant sequence similarity, suggesting MINAS-60 is conserved (Figure 2d). To determine whether mouse MINAS-60 is also translated, a mammalian expression vector contains the 5'UTR of mouse RBM10

222 transcript variant 1 through the stop codon of the putative mouse MINAS-60

223 homolog was transfected into 3T3 cells, followed by immunostaining. As shown

224 in Figure 2e, over-expressed mouse MINAS-60 localized to the nucleolus,

225 comparable to human MINAS-60. Taken together, these results indicate that

226 MINAS-60 is endogenously expressed, cell-cycle regulated and conserved from

227 humans to mouse.

\section{MINAS-60 is associated with nucleolar LSU assembly factors}

Because many SEPs characterized to date bind to and regulate other proteins ${ }^{42}$, we performed a co-immunoprecipitation (co-IP) with the nuclear lysates from MINAS-60-FLAG KI cells, and HEK 293T cells as a control. Two major bands were specifically present in the $\mathrm{KI}$ co-IP after SDS-PAGE that, upon analysis via label-free quantitative proteomics ${ }^{43}$, yielded 17 proteins enriched $>30$-fold over control. GO analysis of these 17 hits shows that the top 2 enriched biological processes are ribosome biogenesis and ribosomal large subunit (LSU)

238 a more comprehensive picture of MINAS-60-associated proteins, we performed

239 Co-IPs and analyzed the entire molecular weight range with quantitative

240 proteomics; while abundant ribosomal proteins limited depth of detection of other 241 proteins, we observed enrichment of four LSU assembly factors: GTPBP4,

242 MRTO4, BRIX1 and NOP2 (Figure 3a and Supplementary Data 4). Co-IP

243 followed by western blotting confirmed the association of these four factors with 244 MINAS-60 (Figure 3b). The association of MINAS-60 with GTPBP4 and MRTO4 245 did not depend on RNA, because these associations largely survived RNaseA 
246 treatment. In contrast, co-purification of MINAS-60 with BRIX1 and NOP2 was

247 severely diminished after treatment with RNaseA, suggesting that their

248 association with MINAS-60 was likely indirect and RNA-dependent (Figure 3b).

249 We therefore hypothesized that MINAS-60 associates with nucleolar, late pre-

25060 s particles containing GTPBP4 and MRTO4 to regulate ribosome

251 biogenesis ${ }^{18,44}$.

252

253 To determine whether MINAS-60 associates with high molecular weight pre-60S

254 particles containing GTPBP4 and MRTO4, we performed sucrose gradient

255 fractionation of nuclear extracts of HEK 293T cells stably expressing epitope-

256 tagged MINAS-60, followed by western blotting analysis. As shown in Figure 3c,

257 a subpopulation of over-expressed MINAS-60 co-sedimented with ribosome

258 assembly factors in high-molecular weight fractions coincident with pre-ribosomal

259 particles. Combined with our co-IP and immunofluorescence data, these results

260 are consistent with a role for MINAS-60 in pre-ribosomal particles.

\section{MINAS-60 inhibits protein synthesis and cell proliferation}

263 We hypothesized that MINAS-60 could regulate ribosome biogenesis via its association with GTPBP4 and MRTO4. Ribosome biogenesis is required for protein synthesis, which promotes cell growth and proliferation. As a result, 266 ribosome biogenesis is commonly upregulated in cancer cells ${ }^{21,45}$. We reasoned 267 that, if MINAS-60 regulates ribosome biogenesis, its absence should result in 268 changes to protein synthesis and cell proliferation. In order to test this 269 hypothesis, we required a system to query the function of MINAS-60 independent 270 of RBM10, despite their co-encoding on the same transcript. To this end, we 271 knocked down (KD) RBM10 in HEK 293T cells with two different shRNAs, which 272 silence the entire mRNA and, therefore, both proteins (RBM10 and MINAS-60).

273 To deconvolute phenotypic effects specific to MINAS-60 in the KD, as well as to 274 exclude off-target effects of shRNA, we generated rescue cell lines stably 275 expressing MINAS-60 (Rescue_MINAS-60) or RBM10 (Rescue_RBM10) on the

$276 \mathrm{KD}$ background. $\mathrm{qRT}-\mathrm{PCR}$ and western blotting analysis revealed that RBM10 
277 mRNA is efficiently silenced by both shRNAs, and the KD cells successfully re-

278 express MINAS-60 or RBM10 after rescue (Supplementary Figure 5). Noting that

279 the RBM10 rescue construct could be subject to leaky translation to produce both

280 RBM10 and MINAS-60, we also rescued the KD with an RBM10 construct

281 bearing an A398TG to TAA mutation that eliminates the first MINAS-60 start

282 codon while preserving RBM10 translation (Supplementary Figure 5).

To test the effect of MINAS-60 expression on cellular protein synthesis, we labeled nascent peptides in the control, RBM10 KD, Rescue_MINAS-60, Rescue_RBM10 and Rescue_RBM10(A398TG-TAA) cell lines with puromycin followed by anti-puromycin western blotting ${ }^{46}$. As shown in Figure $3 \mathrm{~d}$ and 288 Supplementary Figure 6a, depletion of the entire RBM10 mRNA led to a significant increase in global protein synthesis, and this increase was rescued by reintroduction of MINAS-60. Partial rescue by RBM10 reintroduction was also observed, but was not present in the RBM10(A398TG-TAA) rescue cells. Similar results were observed for a second shRNA targeting RBM10 (Supplementary Figures $6 \mathrm{~b}-\mathrm{c}$ ). Taken together, these results indicate that MINAS-60 plays an unusual role in ribosome biogenesis to downregulate global protein synthesis. At the same time, RBM10 is unlikely to play a role in this process. We speculate that the partial rescue observed with RBM10 could have been due to leaky

Because protein synthesis, cell growth and proliferation are linked ${ }^{16,21}$, we asked whether MINAS-60 regulates cell proliferation. As shown in Figure 3e, RBM10 depletion led to a significant increase in cell proliferation, consistent with a published report using HeLa cells ${ }^{29}$. Remarkably, this increase was rescued by reintroduction of MINAS-60 alone, and partially rescued by RBM10 reintroduction. Similar results were observed for a second shRNA targeting RBM10 (Supplementary Figure 6d). These results are consistent with a model in which MINAS-60 inhibits ribosome biogenesis, subsequently downregulating cellular protein synthesis and cell proliferation. 
MINAS-60 inhibits the export of pre-60S ribosome subunits

Eukaryotic ribosome biogenesis can be divided into sequential processes ${ }^{47}$ : prerRNA transcription; chemical modification and processing of the pre-rRNA, both of which occur in the nucleolus; folding, assembly and maturation of the preribosomal subunits in the nucleolus and nucleus; and export and final maturation of ribosome subunits in the cytoplasm. Because MINAS-60 co-purified with proteins involved in various steps of LSU biogenesis, we wished to determine the step in this process that it regulates.

To determine whether MINAS-60 controls pre-rRNA transcription, we performed qRT-PCR targeting the primary pre-rRNA (47S/45S/30S) using previously published primers ${ }^{46}$ in lysates from control, RBM10 KD, Rescue_MINAS-60 and Rescue_RBM10 cell lines. No significant differences in primary pre-rRNA were observed, suggesting that MINAS-60 does not regulate pre-rRNA transcription (Supplementary Figures 7a-b). To determine whether MINAS-60 regulates the processing of LSU pre-rRNA, we performed northern blotting analysis with the control, RBM10 KD, Rescue_MINAS-60 and Rescue_RBM10 cell lines using a probe complementary to ITS2, which detects all LSU pre-rRNA processing products, including 41S, 32S and $12 \mathrm{~S}$ pre-rRNAs ${ }^{46}$. As shown in Supplementary Figure 7c, no significant differences were observed between these cell lines,

We therefore examined the role of MINAS-60 in LSU assembly and export. To determine whether MINAS-60 regulates nucleocytoplasmic export of the pre-60S ribosomal subunit, we quantified the ratio of nuclear vs. cytoplasmic RPL29-

334 GFP ${ }^{48}$ stably expressed in the control, RBM10 KD, Rescue_MINAS-60 and 335 Rescue_RBM10 cell lines. As shown in Figure 4a and Supplementary Figure 8a, 336 depletion of RBM10 decreased the ratio of nuclear to cytoplasmic RPL29-GFP, 337 which can be rescued by reintroduction of MINAS-60, and partially rescued by 338 RBM10 reintroduction, likely due to leaky MINAS-60 translation. Similar results 
were observed for a second shRNA targeting RBM10 (Figure 4a, right, and Supplementary Figures $8 b-c)$. These observations are consistent with the inhibition of cytoplasmic export of pre-60S subunits by MINAS-60. As a control, to determine whether MINAS-60 regulates nucleocytoplasmic export of the pre$40 S$ ribosomal subunit, we quantified the ratio of nuclear vs. cytoplasmic RPS2GFP48 stably expressed in the control, RBM10 KD, Rescue_MINAS-60 and Rescue_RBM10 cell lines. As shown in Supplementary Figure 9, no significant changes were observed between these cell lines, suggesting MINAS-60 does not regulate the assembly or export of $40 \mathrm{~S}$ ribosomal subunits.

The observation of upregulated pre-60S export in the absence of MINAS-60 predicted that the same conditions should lead to an increase in mature cytoplasmic $60 \mathrm{~S}$ ribosomal subunits. To test this, we performed cytoplasmic polysome profiling. As shown in Figures $4 \mathrm{~b}-\mathrm{c}$, knockdown of RBM10 increased the ratio of cytoplasmic $60 S / 40 S$ ribosome subunits, and this increase can be rescued by reintroduction of MINAS-60, and partially rescued by RBM10. Similar results were observed for a second shRNA targeting RBM10 (Figure 4c, right and Supplementary Figure 10). Taken together, these results suggest that MINAS-60 specifically decreases mature large ribosomal subunits by negatively regulating the assembly or export of pre-60S particles.

\section{MINAS-60 inhibits the late-stage assembly of pre-60S}

Lastly, we speculated that MINAS-60 functions as a checkpoint inhibitor in pre605 assembly prior to export from the nucleus, which would suggest that the protein composition of LSU precursors should change in cells lacking MINAS-60. MRTO4 and BRIX1 are LSU biogenesis factors present in multiple intermediateto-late, or early-to-intermediate, pre-60S particles, respectively, and we examined their interactomes as a readout of changes in pre-60S protein composition in the presence or absence of MINAS-60 (Figure 5a) ${ }^{18}$. We stably expressed MRTO4-FLAG or BRIX1-FLAG, in control or RBM10 KD HEK 293T cells to enable affinity purification of pre-ribosomal particles, followed by 
370 quantitative proteomics and western blotting. We detected statistically significant

371 increases in several late LSU assembly factors co-purified with MRTO4 in

372 RBM10 KD cells (Figure 5b, Supplementary Data 5). These increases were

373 further confirmed by western blotting (Figure $5 c$ ). Similar results were observed

374 for BRIX1 co-IP, though fold changes were smaller (Figures $5 \mathrm{~d}-\mathrm{f}$, Supplementary

375 Data 6). These results suggest that remodeling of pre-60S particles toward more

376 mature stages occurs in the absence of MINAS-60, consistent with MINAS-60

377 acting as an inhibitor for LSU assembly and export.

\section{Discussion}

380 In this work, we developed a bio-orthogonal strategy for the direct detection of unannotated nascent or stress-induced alt-proteins. BONCAT has been powerfully applied in prior studies to examine nascent protein synthesis, particularly in neurons ${ }^{30}$, but standard BONCAT workflows, like many proteomics protocols, include column or gel resolution steps that de-enrich small proteins and peptides, and are therefore refractory to detection of microproteins and altproteins. For the first time in this work, we followed azidohomoalanine labeling with small protein enrichment using a previously reported C8 column strategy ${ }^{31}$, coupled with direct on-bead capture to eliminate the need for column-based removal of excess biotin probe molecules, which also remove small proteins.

390 Interfacing this BONCAT-based chemoproteomic pipeline with our platform technology for unannotated microprotein and alt-protein detection, we identified 22 alt-proteins undergoing active synthesis in HEK 293T cells. For six selected alt-proteins, we verified their translation, one of which, alt-CNPY2, is likely posttranscriptionally upregulated by DNA damage stress. We furthermore propose

396 that actively synthesize this protein during $S$ phase of the cell cycle, consistent 397 with its coordinated synthesis with the ribosome biogenesis machinery. Taken 398 together, these results show that our method is able to detect unannotated alt399 proteins expressed during the cell cycle and cellular stress, and that these 400 proteins may play important roles in the cell. In the future, an important next step 
401 will be to characterize the biological role of alt-CNPY2, and to expand this

402 strategy to additional cellular stress conditions.

We then conducted a focused functional study of nucleolar MINAS-60, loss of which promotes the late-stage assembly of pre-60S and the export of pre-60S particles into cytoplasm, leading to increases in cytoplasmic 605 ribosomal subunits, global protein synthesis, and cell proliferation (Figure 6). The MINAS60 alt-protein is therefore a repressor of LSU biogenesis. In contrast to smORFencoded proteins, few alt-ORFs nested within canonical protein CDS have been defined in molecular detail. This work, combined with previous literature ${ }^{7,49}$, expands the recent finding that a single human transcript can encode overlapping, sequence-independent, yet functionally related proteins. Importantly, the RBM10 gene plays important roles in human physiology and disease. RBM10 (RNA Binding Motif 10) is an RNA binding protein that regulates alternative pre-mRNA splicing ${ }^{29,50}$. Null mutations in the RBM10 gene are found in patients with TARP syndrome ${ }^{51}$, an X-linked inherited pathology associated with malformation of multiple organs and significant early-life mortality. The RBM10 gene was also found to be among the most frequently mutated genes in lung adenocarcinoma samples, and RBM10 inhibits cancer cell proliferation ${ }^{52}$. The finding that RBM10 dually encodes MINAS-60, which also downregulates cell proliferation via repression of ribosome biogenesis, opens the question of whether this alt-protein contributes to RBM10 mutation associated disease phenotypes, analogous to alt-FUS, which forms pathogenic cytoplasmic aggregates similar to those caused by the co-encoded FUS protein ${ }^{3}$.

Several studies have suggested that as many as $50 \%$ of multicistronic human genes encode proteins that directly interact to form complexes ${ }^{9,53}$. In contrast, the alt-protein alt-RPL36 can regulate the same pathway (translation) as the canonical protein co-encoded with it (RPL36), without directly interacting with each other ${ }^{8}$. In this work, we show that MINAS-60 and RBM10 exhibit comparable antiproliferative cellular effects but act via independent pathways: 
432 ribosome biogenesis vs. alternative pre-mRNA splicing, respectively. While it

433 remains possible that RBM10 regulates splicing of some nucleolar proteins,

434 RBM10 reintroduction did not rescue the protein translation defect observed in

435 cells lacking RBM10 expression in this study. Based on these results we

436 conclude that RBM10 does not significantly contribute to ribosome biogenesis.

437 We therefore speculate that selective pressure for co-encoding multiple

438 functional proteins in multicistronic human genes may act at the level of cellular

439 fitness, and direct interaction between alt-proteins and canonical proteins is only

440 one mechanism among many by which the fitness effects of co-encoded proteins

441 may be optimized. Another possible mechanism is that, since alt-ORFs may

442 represent protogenes ${ }^{54}$, de novo acquisition of a novel alt-ORF within a protein

443 coding gene might lead to positive selection if both the alt-ORF and canonical

444 protein coding sequence increase cellular fitness under the same conditions,

445 without necessarily acting on the same pathway or process. This broadens the

446 current models of multicistronic human gene function ${ }^{9}$.

448 To our knowledge, MINAS-60 is only the second human microprotein or alt449 protein validated to localize to the nucleolus to date, and the first reported to 450 regulate ribosome biogenesis. A previous study identified nucleolar microprotein 451 C11 orf98, which interacts with nucleolar proteins NPM1 and NCL, but no role for 452 this factor in ribosome biogenesis has been investigated ${ }^{33}$. In this work, we 453 reported MINAS-60 as the first protein that specifically inhibits LSU biogenesis,

454 and we suspect that it functions as a mammal-specific checkpoint to ensure 455 correct pre-60S assembly prior to nuclear export. Supporting the hypothesis that 456 MINAS-60 has arisen in higher eukaryotes, the RBM10 gene is not conserved in 457 yeast, though we cannot yet rigorously conclude that a functional homolog of 458 MINAS-60 is absent in lower eukaryotes. Direct experimental evidence will be 459 required to test the hypothesis that MINAS-60 could represent a control point for 460 ribosome biogenesis that is unique to mammalian cells. 
462 Interestingly, another mammal-specific, putative LSU biogenesis inhibitor has 463 recently been identified. A structural study revealed that an unidentified protein 464 (protein $\mathrm{X}$ ) is positioned to block the incorporation of nuclear export factor NMD3 465 into the late pre-60S particle, hypothetically suppressing pre-60S export into the 466 cytoplasm ${ }^{44}$. Protein $X$ binds in the immature peptidyl transfer center and directly 467 contacts GTPBP4 as well as helix 89 of the rRNA. Importantly, protein X was 468 specifically observed in cryo-electron microscopy structures of human pre-60S 469 particles and has not been detected in structures of yeast LSU precursors ${ }^{44}$.

470 Based on the observations that 1) both MINAS-60 and protein $\mathrm{X}$ likely function as 471 repressors of LSU biogenesis at the late assembly and export stage; and 2) both 472 MINAS-60 and protein X associate with GTPBP4, we speculate that MINAS-60 473 functions by a similar mechanism to protein $\mathrm{X}$. It is possible that protein $\mathrm{X}$ and 474 MINAS-60 are the same protein, and that protein $X$ could not be identified due to 475 its absence from the UniProt database; however, efforts to model MINAS-60 476 sequences into the reported structures remain inconclusive to date. It is therefore 477 also possible that multiple previously unknown mammalian proteins regulate LSU 478 biogenesis, suggesting that regulation of ribosome biogenesis may be more 479 complex in human cells than in yeast.

Why, then, might MINAS-60 have arisen in mammalian cells? In order to achieve a balance between cellular growth requirements and energy-intensive ribosome production ${ }^{15}$, and to ensure that improperly assembled ribosome subunits do not lead to mistranslation ${ }^{23}$, ribosome biogenesis needs to be precisely monitored in cells. For example, the Rio1-Nob1-Pno1 network establishes a checkpoint to safeguard against the release of immature $40 \mathrm{~S}$ subunits into translating ribosomes ${ }^{55}$. However, while hundreds of proteins required for ribosome biogenesis in human cells have now been identified ${ }^{56}$, few repressors of ribosome biogenesis have been reported, and the ones that have been identified predominately act on pre-ribosomal RNA transcription ${ }^{57-59}$. LSU biogenesis

492 the peptidyl transferase center (PTC). Our data lead us to speculate that MINAS- 
49360 and/or Protein X may act as checkpoints for this process to safeguard cellular 494 energy expenditure and/or faithful PTC formation. This hypothesis could be 495 tested by examining the effects of MINAS-60 on cellular fitness under nutrient 496 stress, as well as on the fidelity and accuracy of global protein translation.

497

498 In conclusion, this study, combined with previous literature ${ }^{13}$, demonstrates the 499 power of chemoproteomics to reveal alt-proteins that are regulated and/or 500 functional in important cellular processes including DNA damage and ribosome 501 biogenesis, and reveals an entirely new regulatory node of the ribosome 502 biogenesis pathway, meriting further development of chemical tools to enrich and 503 identify functional alt-proteins.

504

505

506 
a

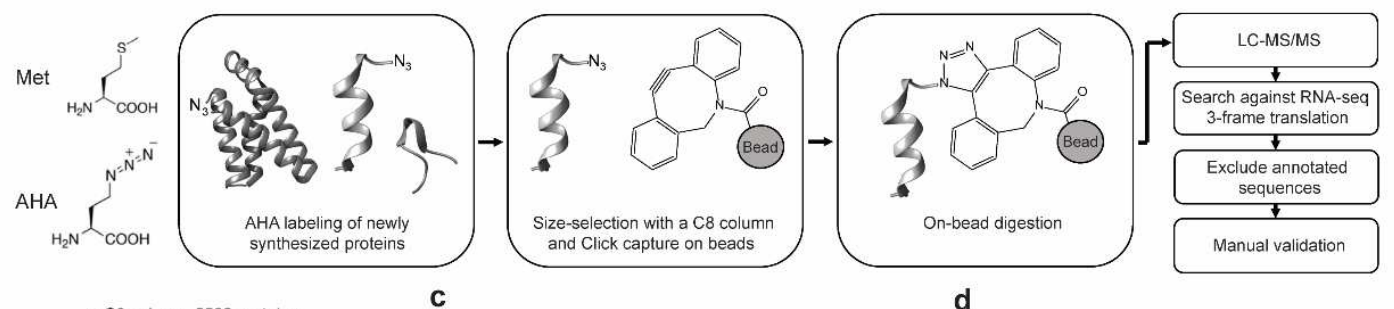

b

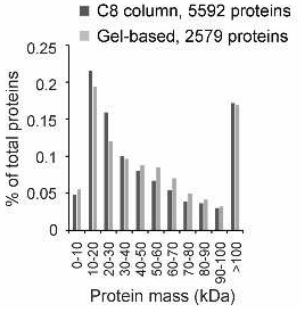

d
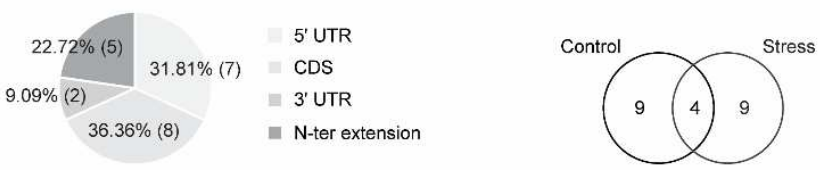

e

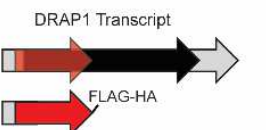

PRR3 Transcript Variant 2
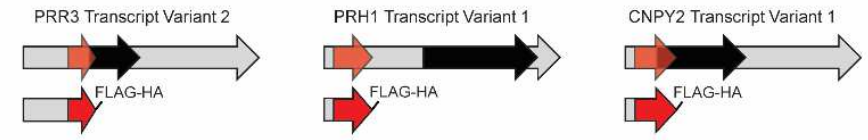

CACTIN Transcript Variant 1

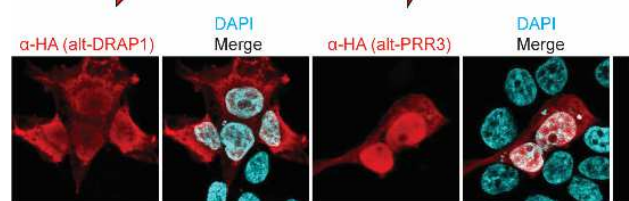

DAPI

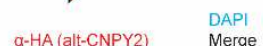

DAPI
Merge
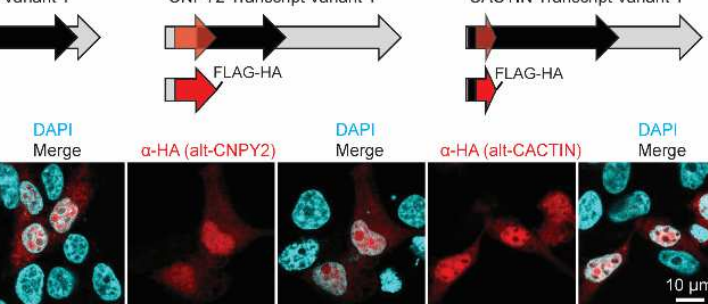

g

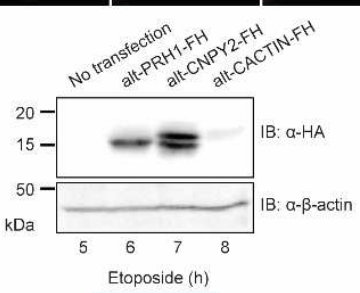

h
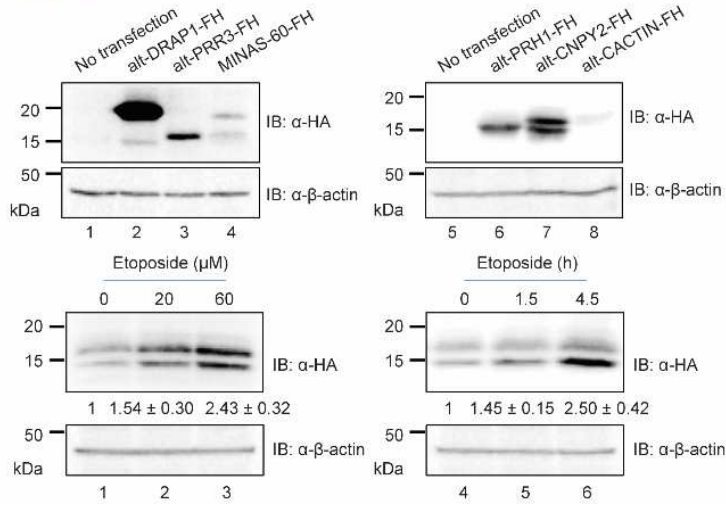

Figure 1. BONCAT-based chemoproteomic identification of newly synthesized alt-proteins. a Schematic workflow for BONCAT-based

chemoproteomic analysis of nascent alt-proteins. b Size distribution of canonical,

511 annotated proteins detected using C8 column-based selection (black) and our

512 previously reported ${ }^{60}$ gel-based selection (gray). c Distribution of locations of

513 identified alt-proteins relative to the annotated coding sequence (CDS). d Venn

514 diagram of alt-proteins identified under control or stress conditions. e, f Top: a

515 schematic representation of human DRAP1 transcript, PRR3 transcript variant 2,

516 PRH1 transcript variant 1, CNPY2 transcript variant 1, CACTIN transcript variant

517 1, or RBM10 transcript variant 1 (c); light gray arrow, 5' and 3' untranslated 
518 regions (UTR); red, alternative open reading frame (alt-ORF) coding sequence;

519 black, annotated coding sequence. Middle: a schematic representation of the

520 expression constructs containing the complete 5'UTR and the alt-ORF of the

521 transcript indicated above, with a dual FLAG and HA tag appended to the C-

522 terminus of the alt-protein. Bottom: HEK 293T cells transfected with the

523 expression construct indicated (middle) were immunostained with anti-HA (red),

524 DAPI (cyan), and anti-fibrillarin (green, c). Scale bar, $10 \mu \mathrm{m}$. Data are

525 representative of three biological replicates. $g$ HEK 293T cells transfected with

526 the expression construct (middle) were immunoblotted (IB) with antibodies

527 indicated to the right, with untransfected (no transfection) HEK 293T cells as a

528 control. Data are representative of three biological replicates. h HEK 293T cells

529 transfected with the alt-CNPY2 expression construct were treated with increasing

530 amounts of etoposide or vehicle for $2 \mathrm{~h}$ (left), or with $60 \mu \mathrm{M}$ etoposide for

531 different times or vehicle (right), followed by western blotting with antibodies

532 indicated to the right. Quantitative analysis $(N=3)$ of the western blot signal of

533 alt-CNPY2-FLAG-HA are indicated at the bottom. 
a

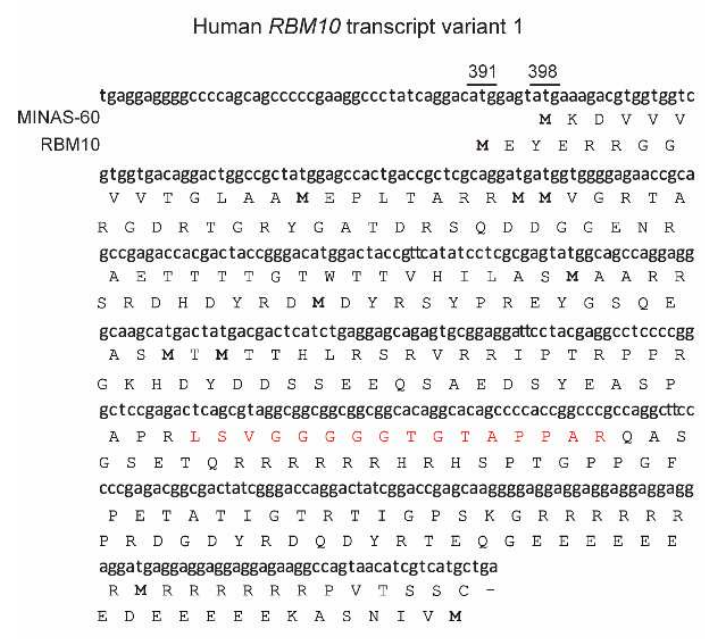

b

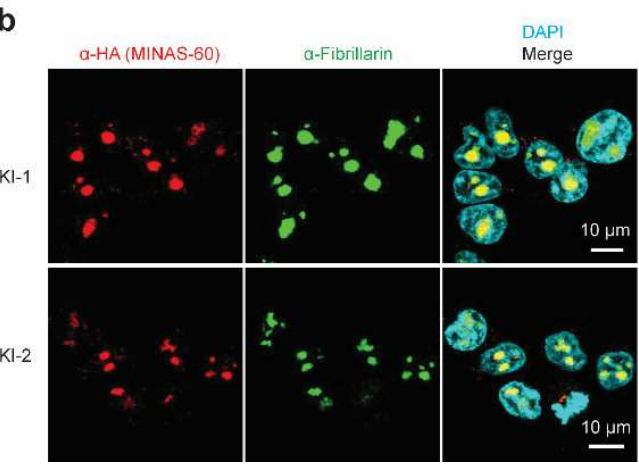

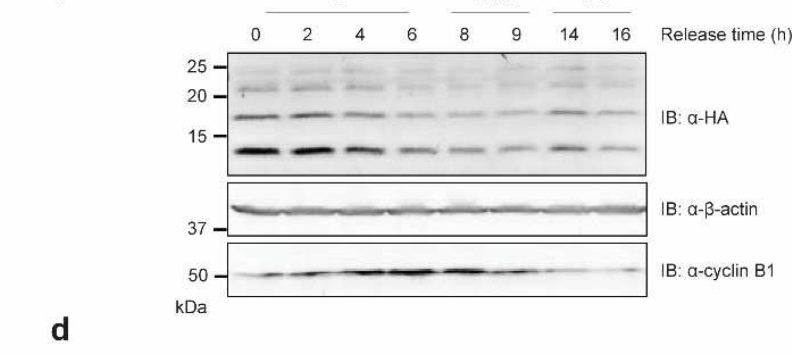

Mus musculus MKDEVVVVTGLAAMEPLIVHRMIVERTAAGTMIIETWTTAHTHESMAVRRASMSMTTHLK 60 Bos taurus MKDEGDVETGLAVTEPPIVHRMTVGKTVAGIMTTGTWTTAHIPASTAARRASMTMMTHLR 60 Homo sapiens MKDVWVVTGLAAMEPLTARRMMVGRTAAETTTTGTWTTVHILASMAARRASMTMTTHLR 60 Macaca mulatta MKDVVVVVTGLGAMEPLTARRMMVGRTAAETTTTGTWTTVHIPASTAARRASTTMTTHLR 60 $* * * * * * \ldots * * \quad .: * * * * * . * \quad * * * * . * * * * * * * * * * * *$
SKVQRIPTRPPRAPRLSVGGGGGTGTVPLARQASPETATIGTRTIGPSKGRRRRRRMRRR 120 SRVQRIPTRPPRAPRLSVGGGGGTGTALPARQASPETATIGTRTIGPSKGRRRRRRRRRR 120 SRVRRIPTRPPRAPRLSVGGGGGTGTAPPARQASPETATIGTRTIGPSKGRRRRRRRMRR 120 SRVRRIPTRPPRAPRLSVGGGGGTGTAPPARRASPETATIGTRTIGPSKGRRMRRRRMRR 120

RRRRPVTSSC 130 RRRRPVTSSC 130 RRRRPVTSSC 130 RRRRPVTSSC 130

e

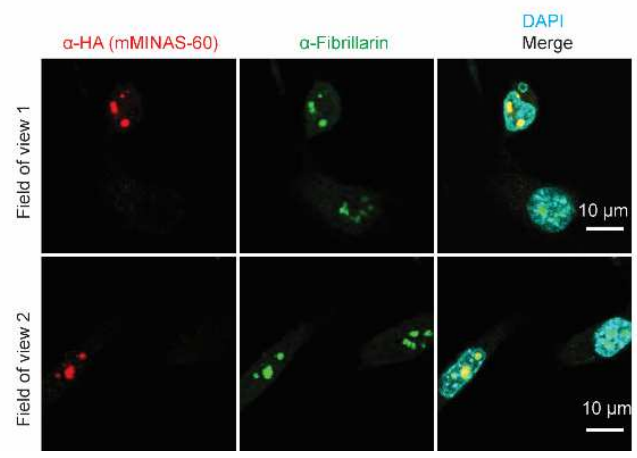

Figure 2. MINAS-60 is endogenously expressed, cell-cycle regulated and conserved in mouse. a Shown is the cDNA sequence of human RBM10 transcript variant 1 with the protein sequences of MINAS-60 and RBM10 indicated below. The start codons of RBM10 (391) and MINAS-60 (398) are numbered above the cDNA sequence. Highlighted in red is the tryptic peptide of MINAS-60 detected by LC-MS/MS in this study. $\mathbf{b}$ Immunostaining of two MINAS-60 knock-in (KI) HEK 293T cell lines with anti-HA (red), anti-fibrillarin (green), and DAPI (cyan). Scale bar, $10 \mu \mathrm{m}$. Data are representative of three biological replicates. c Western blotting analysis of synchronized MINAS-60 KI cells released from the G1/S boundary at the indicated time points with antibodies indicated on the right. Data are representative of three biological replicates. d RBM10 mRNAs from Mus musculus (NM_145627.3), Bos taurus (NM_001206693.1), and Macaca mulatta (XM_015127275.2) were obtained from NCBI nucleotide database, then translated in the $+1,+2$ and +3 frames using the 
550 Expasy translate tool. Cognate start codons in-frame with sequences

551 homologous to human MINAS-60 were identified in the 5'UTR of each transcript

552 in order to predict the full-length sequence of hypothetical MINAS-60 homologs.

553 Shown is the ClustalW2 alignment of predicted MINAS-60 protein sequences. e

554 3T3 cells transfected with the mouse MINAS-60 (mMINAS-60) expression

555 construct were immunostained with anti-HA (red), anti-fibrillarin (green), and

556 DAPI (cyan). Scale bar, $10 \mu \mathrm{m}$. Data are representative of three biological

557 replicates.

558 
a

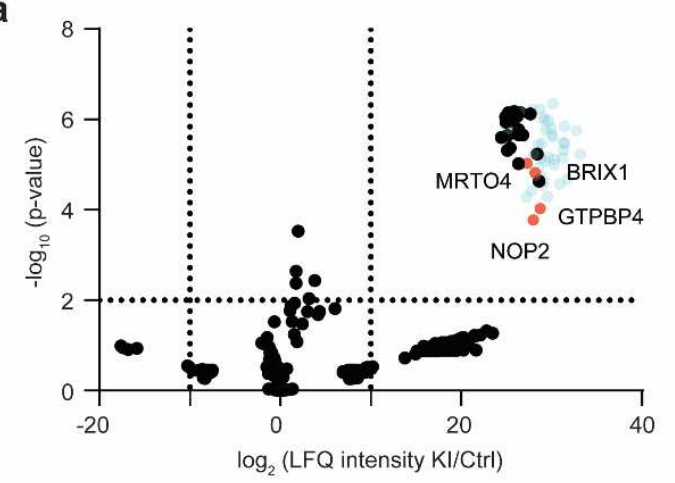

b

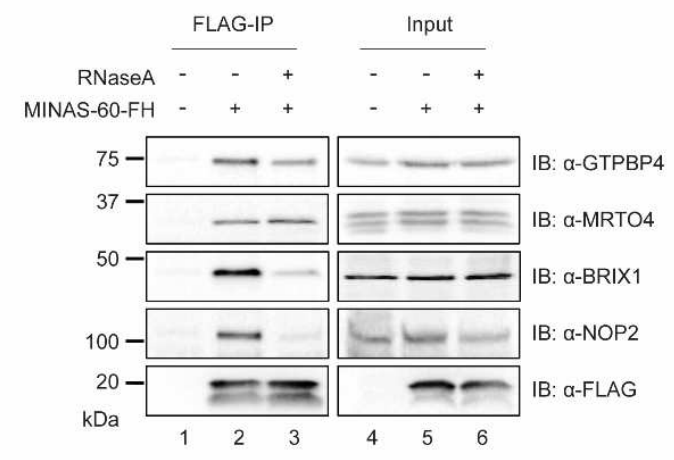

d

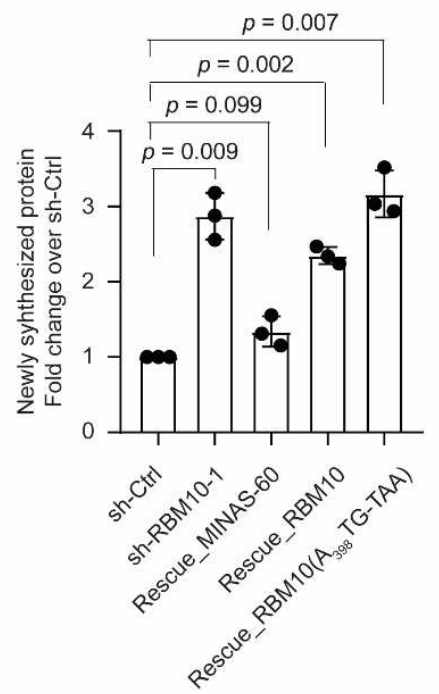

C

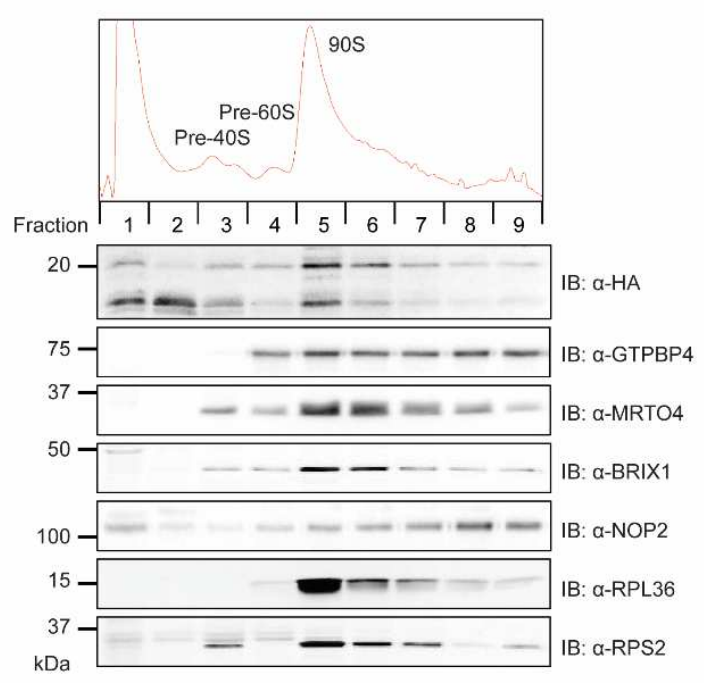

e

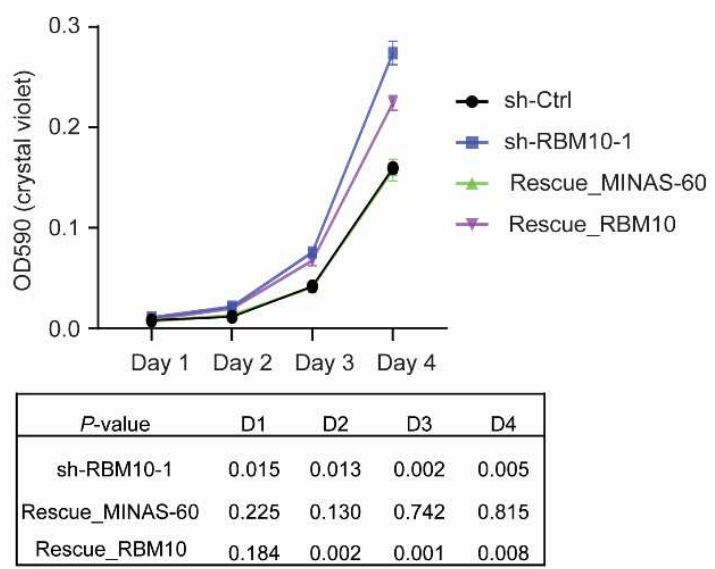

560 Figure 3. MINAS-60 associates with nucleolar LSU assembly factors, downregulates global protein synthesis and cell proliferation. a Volcano plot of quantitative proteomics $(N=3)$ of anti-FLAG pulldown from MINAS-60 KI $(\mathrm{KI})$

563 or control (Ctrl) HEK 293T nuclear lysates. Ribosomal proteins are indicated in 564 blue. Enriched LSU assembly factors are indicated in red and gene names are 565 labeled. For complete quantitative proteomics results, see Supplementary Data 566 4. b HEK 293T cells were transfected with MINAS-60-FLAG-HA (MINAS-60-FH, 
567 lanes 2 and 3) or vehicle (lane 1), and immunoprecipitation (FLAG-IP) was 568 performed in absence (lanes 1 and 2) or presence (lane 3) of RNaseA, followed 569 with immunoblotting (IB) with antibodies indicated on the right. Cell lysates (4\%) 570 before IP (input, lanes 4-6) were used as loading controls. c Top: Sucrose571 gradient sedimentation analysis of nuclear lysates containing ribosome precursor

572 complexes (pre-40S, pre-60S and 90S pre-ribosome) from HEK 293T cells stably 573 expressing MINAS-60-FLAG-HA. Bottom: Western blot analysis of fractions 574 numbered at the top with antibodies indicated on the right. $\mathbf{d}$ Image J was used to 575 quantify the relative puromycin incorporation for cells indicated at the bottom 576 relative to sh-Ctrl from three biological replicates. Data represent mean values \pm 577 s.e.m., and significance was evaluated with two-tailed $t$-test. e Growth curve of 578 control (sh-Ctrl), RBM10 knockdown (sh-RBM10-1), rescue with MINAS-60 579 (Rescue_MINAS-60) and rescue with RBM10 (Rescue_RBM10) HEK 293T cells 580 at the indicated number of days $(N=3)$. Data represent mean values \pm s.e.m., 581 and significance was evaluated with two-tailed $t$-test and shown below. 
a

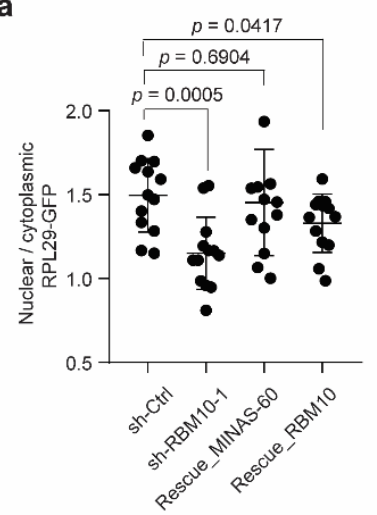

C

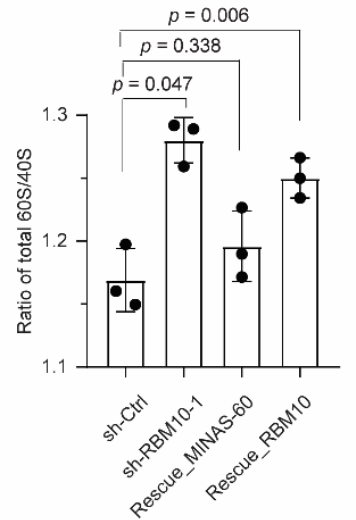

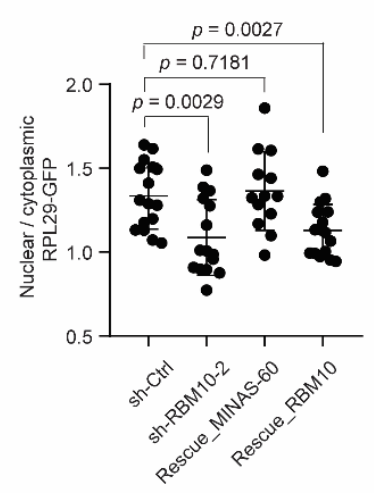

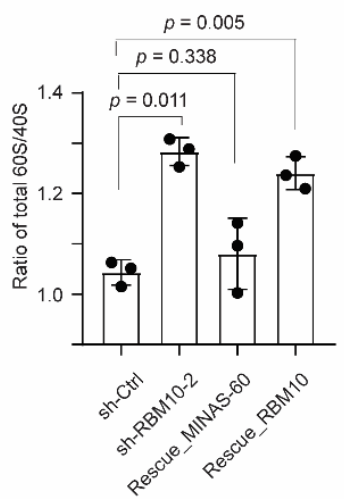

b
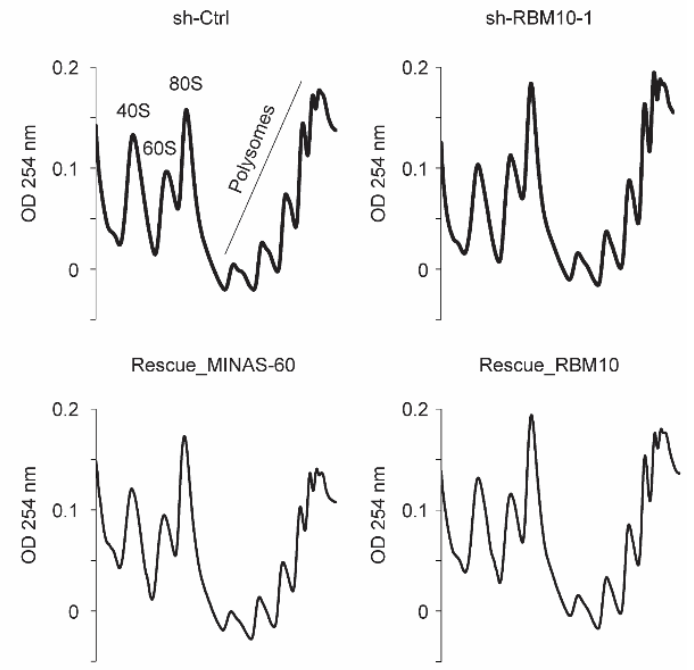

583

584

585

586

587

588

589

590

591

592

593

594

595

596

597

598

599

Figure 4. MINAS-60 inhibits LSU export. a Quantitation of the ratio of RPL29GFP intensity in the nucleus vs. cytoplasm in control (sh-Ctrl), RBM10 knockdown with one of the two shRNAs (sh-RBM10-1 (left), sh-RBM10-2 (right)), rescue with MINAS-60 (Rescue_MINAS-60), or rescue with RBM10 (Rescue_RBM10) HEK 293T cells stably expressing RPL29-GFP. At least 13 fields of view were analyzed, totaling $>350$ cells for each measurement. Data represent mean values \pm s.e.m., and significance was evaluated with two-tailed $t$ test. b Sucrose-gradient sedimentation analysis of ribosomal fractions (40S, 60S, $80 \mathrm{~S}$ and polysomes) of cytoplasmic lysates from control (sh-Ctrl), RBM10 knockdown (sh-RBM10-1), rescue with MINAS-60 (Rescue_MINAS-60) or rescue with RBM10 (Rescue_RBM10) HEK 293T cells. Data are representative of three biological replicates. c Quantitation of the ratio of cytoplasmic $60 \mathrm{~S}$ to $40 \mathrm{~S}$ subunits in the cell lines indicated below after sucrose gradient fractionation. The area under each peak was measured using ImageJ following a previously published method ${ }^{61}$. Data represent mean values \pm s.e.m., and significance was evaluated with two-tailed $t$-test. 
a

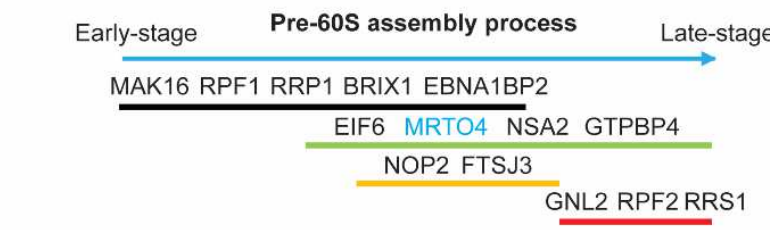

b

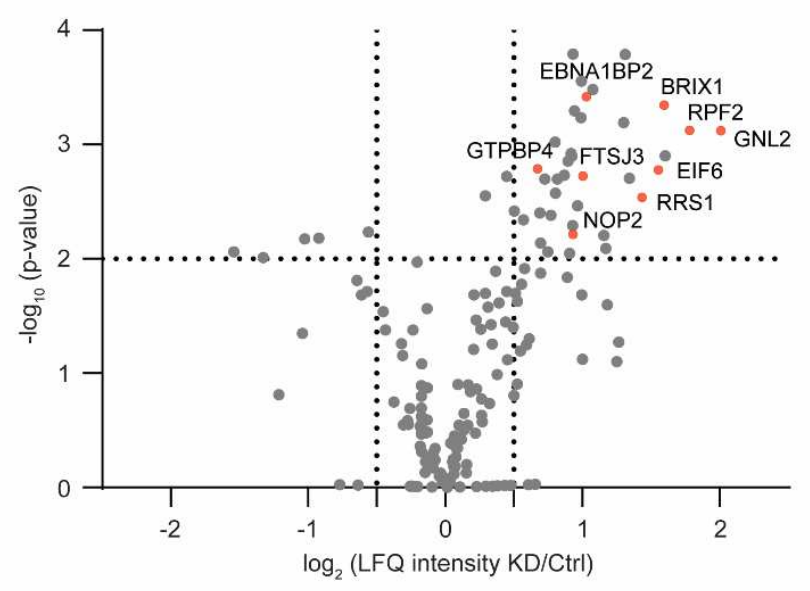

d

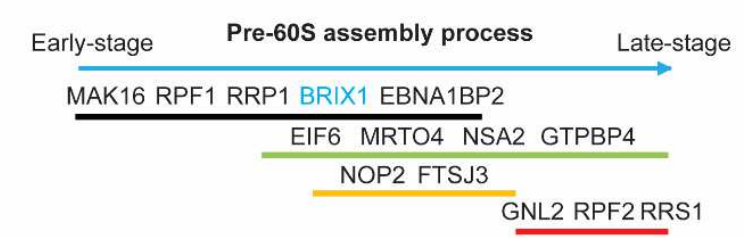

e

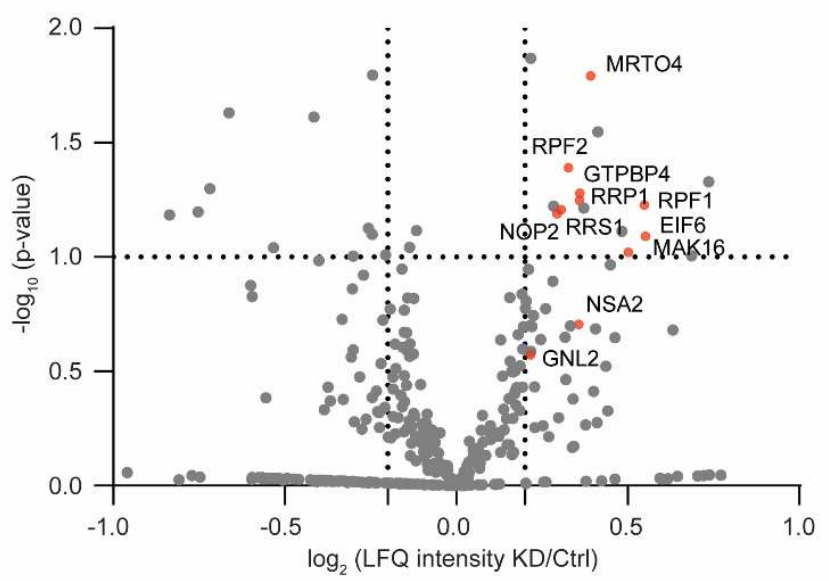

C

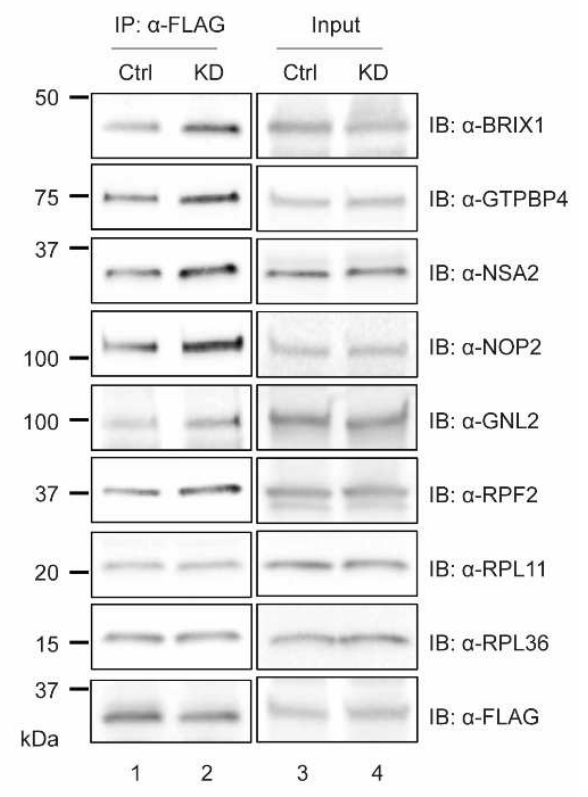

f

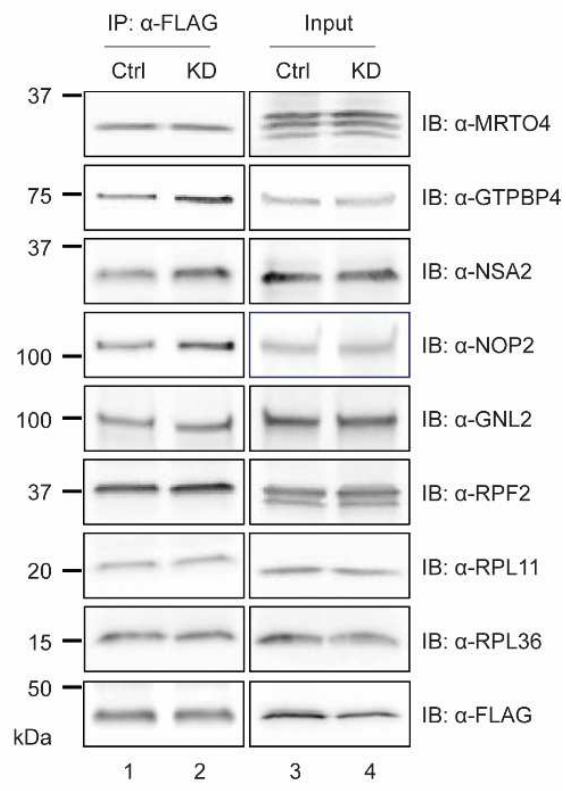

601 Figure 5. RBM10 silencing promotes pre-60S assembly. a, d Schematic

602 representation of pre-60S assembly factors associated with different states

603 based on a pre-60S structure report ${ }^{18}$. The bait protein MRTO4 (a) and BRIX1

604 (d) is indicated in blue. b, e Volcano plot of quantitative proteomics $(N=4$ (b), $N$

$605=5(\mathbf{e}))$ of MRTO4-FLAG (b) or BRIX1-FLAG (e) pulldown from HEK 293T cells 
606 stably expressing the bait protein and control shRNA (Ctrl), or the bait protein 607 and RBM10 shRNA (KD), to quantify relative changes in the bait protein co-IP of 608 LSU assembly factors in RBM10 KD over control HEK 293T cells. Increased 609 assembly factors are indicated in red and gene names are labeled. For complete 610 quantitative proteomics results, see Supplementary Datas 5 and 6. c, f MRTO4 611 FLAG-IP (c) or BRIX1-FLAG-IP (f) and western blotting with antibodies indicated 612 on the right using the two cell lines described above. Cell lysates (4\%) before IP 613 (input) were used as the loading control. Data are representative of three 614 biological replicates.

615 

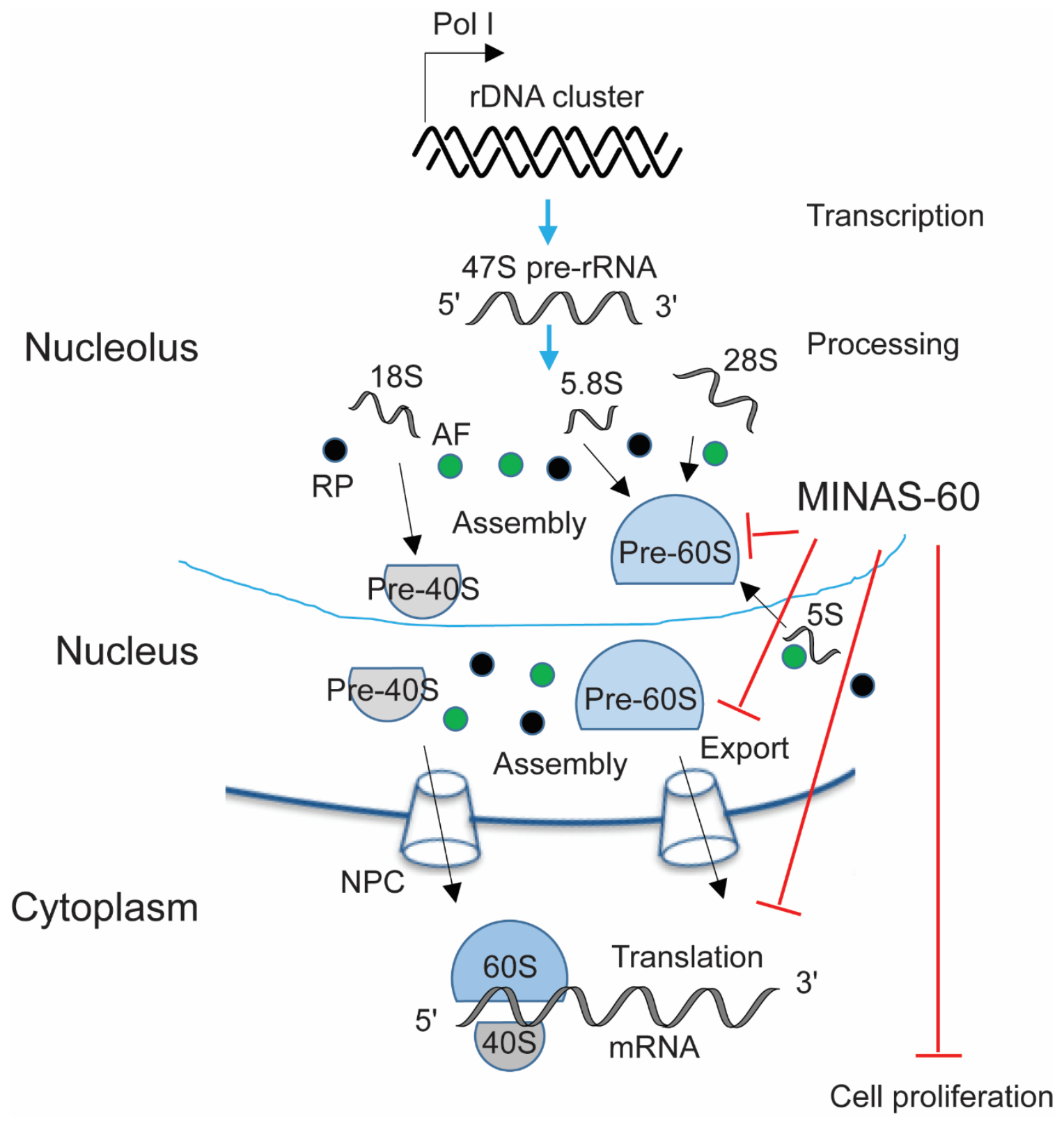

Figure 6. Model of MINAS-60 regulatory pathway. MINAS-60 localizes to the

618 nucleolus, where it associates with multiple pre-60S assembly factors, including

619 GTPBP4 and MRTO4, to inhibit the late-stage pre-60S assembly and the export

620 of pre-60S into cytoplasm, consequently downregulating global protein synthesis

621 and cell proliferation.

622 


\section{Online methods}

624 Data analysis. Two-tailed $t$-test were performed using Microsoft Excel or

625 GraphPad Prism, and F-tests were performed to evaluate equal variance 626 between samples.

627 Antibodies. Primary antibodies for western blotting include the following: anti628 FLAG (Sigma, F3165 or Cell Signaling, 14793); anti-HA (Invitrogen, 71-5500); 629 anti- $\beta$-actin (Invitrogen, BA3R); anti-GTPBP4 (Abclonal, A4565); anti-MRTO4 630 (ThermoFisher, 20194-1-AP); anti-NSA2 (Abclonal, A14475); anti-NOP2 (Cell 631 Signaling, 25017); anti-BRIX1 (Abclonal, A14481); anti-RPF2 (Abclonal, 632 A17224); anti-GNL2 (Abclonal, A13191); anti-RPL11 (Cell Signaling, 18163); 633 anti-RPL36 (Bethyl Laboratories, A305065A-M); anti-RPS2 (Invitrogen, PA5634 30160); anti-RBM10 (Abcam, ab72423); anti-puromycin (Kerafast, EQ0001); anti635 GFP (Abcam, ab183734); anti-cyclin B1 (Cell Signaling, 4138).

636 Immunoprecipitation was performed with anti-FLAG M2 affinity gel (Sigma, 637 A2220). Secondary antibodies for western blotting are goat anti-rabbit IgG 638 horseradish peroxidase conjugate (Rockland, 611-1302) and goat anti-mouse 639 IgG horseradish peroxidase conjugate (Rockland, 610-1319-0500). Primary 640 antibodies for immunostaining are rabbit anti-HA (Invitrogen, 71-5500) and 641 mouse anti-Fibrillarin (abcam, ab4566). Secondary antibodies for 642 immunostaining are goat anti-rabbit IgG Alexa fluor 568 (Invitrogen, A11011) and 643 goat anti-mouse IgG Alexa fluor 647 (Invitrogen, A21235).

645 Cloning and genetic constructs. A DNA sequence comprising the full 5'UTR of 646 human $D R A P 1$ transcript, $P R R 3$ transcript variant 2, $P R H 1$ transcript variant 1, 647 CNPY2 transcript variant 1, CACTIN transcript variant 1, or RBM10 transcript 648 variant 1 through the stop codon of the relative alt-protein was amplified by PCR 649 with a dual FLAG and HA epitope tag appended to the 3 ' end of the alt-protein 650 coding sequence from an in-house library of reverse-transcribed HEK 293T 651 cDNAs, then subcloned into pcDNA3. Deletion or mutation constructs of MINAS65260 bearing a dual FLAG and HA tag were generated by ligating PCR products 
653 into BamHI and EcoRI cloning sites in the pcDNA3 vector. For generation of HEK

654293 T cells stably expressing MINAS-60, a dual FLAG and HA tag were

655 appended to the 3 ' end of MINAS-60 by PCR, and the dually tagged coding

656 sequence was then cloned into pLJM1. The cDNA clone expressing RPS2 was

657 purchased from Addgene (a gift from Thomas Tuschl), and the coding sequences

658 of MRTO4, BRIX1 and RPL29 were amplified by PCR from an in-house HEK

$659293 \mathrm{~T}$ cDNA library, then subcloned into pJLM1 for producing lentivirus. RNAi

660 constructs were made by synthesizing oligonucleotides encoding a 21 bp short

661 hairpin RNA that targets RBM10 (shRNA1, CTTCGCCTTCGTCGAGTTTAG;

662 shRNA2, TCCAACGTGCGCGTCATAAAG), then subcloned into pLKO.1. The

663 empty pLKO.1 vector control was purchased from Sigma (SHC001). qPCR

664 primer sequences are provided in Supplementary Table 2.

665

666 Cell culture, lentivirus production and stable cell line generation. HEK 293T

667 cells were purchased from ATCC and early-passage stocks were established in

668 order to ensure cell line identity. Cells were maintained up to only 10 passages.

669 HEK 293T cells were cultured as previously described ${ }^{8}$. To produce lentivirus

670 and generate stable cell lines, HEK 293T cells were co-transfected using

671 polyethyleneimine (Polysciences, 23966) with expression construct in pLJM1,

672 along with pMD2.G and psPAX2, and growth media were replaced after 7-8 h. 48

$673 \mathrm{~h}$ post-transfection, media containing viruses was harvested, filtered through a

$674 \quad 0.45-\mu \mathrm{m}$ filter, and infection was performed by mixing with two volumes of fresh

675 media containing suspended HEK 293T cells. $24 \mathrm{~h}$ post-infection, the growth

676 media was replaced. $48 \mathrm{~h}$ post-infection, stably expressing cells were selected

677 with $4 \mu \mathrm{g} / \mathrm{mL}$ puromycin for 2 days. Early stocks of stable cell lines were

678 established after selection. Stable cell lines were released from puromycin for 2

679 days prior to use in experiments.

680

681 Immunostaining and live-cell imaging. HEK 293T cells were plated on glass

682 coverslips and transfected the next day. Forty-eight hours later, the cells were

683 fixed in $10 \%$ formalin for $15 \mathrm{~min}$ at room temperature (RT), permeabilized with 
PBS containing $0.2 \%(\mathrm{v} / \mathrm{v})$ TritonX-100, then incubated with primary antibodies for $18 \mathrm{~h}$ at $4^{\circ} \mathrm{C}$. After washing with PBS, the cells were incubated with secondary antibodies and DAPI for $1 \mathrm{~h}$ at RT, washed with PBS and mounted with Mowiol (Sigma, 81381) before viewing.

HEK 293T cells stably expressing RPL29-GFP or RPS2-GFP were grown to $70 \%$ confluency on coverslips in 12-well plates. Coverslips were inverted and imaged in pre-warmed DMEM with 10\% FBS, $1 \%$ penicillin-streptomycin in MatTek imaging dishes. Confocal imaging was performed on a Leica SP8 LS confocal microscope with $63 \times$ oil immersion objective with atmosphere-controlled stage at $37^{\circ} \mathrm{C}$. Nuclear/cytoplasmic ratios of RPL29 and RPS2 were measured using the ImageJ Intensity Ratio Nuclei Cytoplasm Tool (RRID:SCR_018573; https://github.com/MontpellierRessources/magerie/imagej_macros_and_scripts/w iki/Intensity-Ratio-Nuclei-Cytoplasm-Tool).

Immunoprecipitation and proteomics. Control HEK 293T cells or MINAS-60 KI cells were grown to $80-90 \%$ confluency in $15 \mathrm{~cm}$ dishes. Cells were harvested and suspended in $1 \mathrm{~mL}$ nuclear isolation buffer (10 mM HEPES-KOH pH 7.4, $100 \mathrm{mM} \mathrm{KCl}, 5 \mathrm{mM} \mathrm{MgCl} 2$ with $0.5 \%$ NP40 and Roche Complete protease inhibitor cocktail tablets (Roche, 11873580001)), and incubated on ice for $10 \mathrm{~min}$, followed by centrifugation at $3,000 \mathrm{~g}, 4^{\circ} \mathrm{C}, 3 \mathrm{~min}$. The nuclear pellets were suspended in $1 \mathrm{~mL}$ lysis buffer (Tris-buffered saline (TBS) with 1\% Triton X-100 and Roche Complete protease inhibitor cocktail tablets), followed with sonication and immunoprecipitation as previously described ${ }^{8}$. After the final wash, elution was in $40 \mu \mathrm{L}$ of $3 \times$ FLAG peptide (Sigma, F4799), at a final concentration of 100 $\mu \mathrm{g} / \mathrm{mL}$ in lysis buffer at $4^{\circ} \mathrm{C}$ for $1 \mathrm{~h}$. The eluted proteins were subjected to SDSPAGE separation prior to LC-MS/MS analysis.

Gel slices, containing either resolved protein bands or entire lanes, were digested with trypsin at $37^{\circ} \mathrm{C}$ for $14-16 \mathrm{~h}$. The resulting peptide mixtures were extracted from the gel, dried, subjected to ethyl acetate extraction to remove 
715 residual detergent, de-salted with peptide cleanup C18 spin column (Agilent

716 Technologies, 5188-2750), then re-suspended in $35 \mu \mathrm{L} 0.1 \%$ formic acid (FA),

717 followed by centrifugation at $21,130 \mathrm{~g}, 4^{\circ} \mathrm{C}, 30 \mathrm{~min}$. A $5 \mu \mathrm{L}$ aliquot of each

718 sample was injected onto a pre-packed column attached to a nanoAcquity UPLC

719 (Waters) in-line with a Thermo Scientific ${ }^{\mathrm{TM}} \mathrm{Q}$ Exactive $^{\mathrm{TM}}$ Plus Hybrid

720 QuadrupoleOrbitrap ${ }^{\mathrm{TM}}$ mass spectrometer (Thermo Scientific) and a 130-min

721 gradient was used to further separate the peptide mixtures as follows (solvent A:

$7220.1 \% \mathrm{FA}$; solvent $\mathrm{B}$ : acetonitrile ( $\mathrm{ACN}$ ) with $0.1 \% \mathrm{FA})$ : Isocratic flow was

723 maintained at $0.1 \mu \mathrm{L} / \mathrm{min}$ at $1 \% \mathrm{~B}$ for $40 \mathrm{~min}$, followed by linear gradients from

$7241 \%$ B to $6 \%$ B over $2 \mathrm{~min}, 6 \%$ B to $24 \%$ B over $48 \mathrm{~min}, 24 \%$ B to $48 \%$ B over 5

$725 \mathrm{~min}, 48 \% \mathrm{~B}$ to $80 \%$ B over $5 \mathrm{~min}$. Isocratic flow at $80 \%$ B was maintained for 5

$726 \mathrm{~min}$, followed by a gradient from $80 \% \mathrm{~B}$ to $1 \% \mathrm{~B}$ over $5 \mathrm{~min}$, and isocratic flow at

$7271 \% \mathrm{~B}$ was maintained for $10 \mathrm{~min}$. The full MS was collected over the mass range

728 of $300-1,700 \mathrm{~m} / \mathrm{z}$ with a resolution of 70,000 and the automatic gain control

729 (AGC) target was set as $3 \times 10^{6}$. MS/MS data was collected using a top 10 high-

730 collisional energy dissociation method in data-dependent mode with a normalized

731 collision energy of $27.0 \mathrm{eV}$ and a $1.6 \mathrm{~m} / \mathrm{z}$ isolation window. MS/MS resolution

732 was 17,500 and dynamic exclusion was 90 seconds.

733

734 For identification of alt- and microproteins, ProteoWizard MS Convert was used

735 for peak picking and files were analyzed using Mascot. Oxidation of methionine

736 and $\mathrm{N}$-terminal acetylation were set as variable modifications, and a previously

737 reported $^{12}$ three-frame translation of assembled transcripts from HEK 293T

738 mRNA-seq was used as the database. For co-IP proteomics searches and

739 quantitative analysis, files were analyzed using MaxQuant, oxidation of

740 methionine and $\mathrm{N}$-terminal acetylation were set as variable modifications, and

741 human UniProt plus MINAS-60 was used as the database for searching. For all

742 analysis, a mass deviation of 20 p.p.m. was set for MS1 peaks, and 0.6 Da was

743 set as maximum allowed MS/MS peaks with a maximum of two missed

744 cleavages. Maximum false discovery rates (FDR) were set to $1 \%$ both on peptide

745 and protein levels. Minimum required peptide length was five amino acids. 
746 Protein quantitation was accomplished by calculating the LFQ intensity ratio of KI

747 or KD pulldown to negative control samples using MaxQuant (version 1.6.8.0)

748 with standard parameters.

749

750 Dibenzocyclooctyne (DBCO) bead construction. NHS-activated beads (Pierce, 751 88826) were conjugated to dibenzocyclooctyne-amine (Sigma, 761540) by $90 \mathrm{~min}$ 752 incubation at room temperature with rotation in a saturated $100 \mathrm{mM}$ sodium 753 bicarbonate $(\mathrm{pH} 8.0)$ solution. The beads were then washed and blocked for $2 \mathrm{~h}$ 754 with $3 \mathrm{M}$ ethanolamine according to the manufacturer's instructions. After blocking, 755 the beads were re-suspended in PBS prior to immediate protein conjugation.

756

757

BONCAT (bio-orthogonal non-canonical amino acid tagging). HEK 293T cells were grown to $80-90 \%$ confluency in $15 \mathrm{~cm}$ dishes, treated with the methionine aminopeptidase inhibitor TNP470 (50 nM) for $2 \mathrm{~h}$, then immersed in methionine-free DMEM (Corning, 17-204-Cl) for 30 min before addition of $4 \mathrm{mM}$ AHA (Click Chemistry tools, 1066-1000) in methionine-free DMEM with 10\% FBS. For stress conditions, cells were treated with $4 \mathrm{mM} \mathrm{AHA}$ with simultaneous exposure to $500 \mu \mathrm{M}$ sodium arsenite, $20 \mu \mathrm{M}$ etoposide (Sigma, E1383), or $1 \mathrm{mM}$ DTT. After a $2 \mathrm{~h}$ incubation, the cells were washed twice with cold PBS, harvested, and flash frozen until further processing.

\section{DBCO bead enrichment of AHA-labeled proteins and ERLIC (electrostatic} repulsion hydrophilic interaction chromatography) fractionation. AHAlabeled cells were lysed by boiling in $50 \mathrm{mM} \mathrm{HCl}$ with $0.01 \%$ 2-mercaptoethanol and $0.05 \%$ TritonX-100 for $10 \mathrm{~min}$. The cells were then pelleted at $21,100 \mathrm{~g}, 4^{\circ} \mathrm{C}$ for $30 \mathrm{~min}$, and passed through a $5 \mu \mathrm{m}$ filter before size selection with a C8

772 column (Agilent Technologies, 12102100) essentially as previously reported ${ }^{32}$.

773 The C8 column was pre-conditioned with 1 bed volume of methanol and 2 bed 774 volumes of $0.25 \mathrm{M}$ triethylammonium formate (TEAF, pH 3.0). Then up to 2 bed 775 volumes of cell lysate were loaded on the column, followed by twice washes with 7762 bed volumes of TEAF and elution with 2 bed volumes of ACN:TEAF (1:3). The 
sample was then dried and reconstituted in $300 \mu \mathrm{L}$ PBS. Cell lysates were incubated with DBCO beads for $1 \mathrm{~h}$ at RT. The beads were washed twice with 1 $\mathrm{mL}$ RIPA buffer, once with $1 \mathrm{M} \mathrm{KCl}$, once with $0.1 \mathrm{M}$ sodium carbonate, once with $2 \mathrm{M}$ urea, twice with RIPA buffer, and finally 6 times with PBS. Proteins covalently conjugated to the beads were then subjected to reduction, alkylation and on-bead trypsin digestion according to standard protocols ${ }^{62}$.

Before LC-MS/MS, the digested peptides were fractionated using ERLIC on an Agilent $1100 \mathrm{HPLC}$. Peptides were re-suspended in $55 \mu \mathrm{L}$ of $85 \%$ ACN/0.1\% FA and $50 \mu \mathrm{L}$ was loaded onto a polyWAX LP column $(150 \times 1.0 \mathrm{~mm} ; 5 \mu \mathrm{m} 300 \AA$; PolyLC). Samples were run on an 80 min gradient protocol as follows (Solvent $A$ : $80 \%$ ACN 0.1\% FA; Solvent B: $30 \%$ ACN 0.1\% FA): Isocratic flow was maintained at $100 \% \mathrm{~A}$ at a flow rate of $0.3 \mathrm{~mL} / \mathrm{min}$ for $5 \mathrm{~min}$, followed by a $17 \mathrm{~min}$ linear gradient to $8 \% \mathrm{~B}$, and a $25 \mathrm{~min}$ linear gradient to $45 \% \mathrm{~B}$. Finally, a $10 \mathrm{~min}$ gradient to $100 \%$ B was followed by a $5 \mathrm{~min}$ hold at $100 \%$ B before a $10 \mathrm{~min}$ linear gradient back to $100 \% \mathrm{~A}$, followed by an $8 \mathrm{~min}$ hold at $100 \% \mathrm{~A}$. The digested peptides were separated into 12-15 fractions which were dried and resuspended in $7 \mu \mathrm{L}$ of $3: 870 \%$ FA : $0.1 \%$ TFA before LC-MS/MS analysis.

Generation of knock-in cell lines. MINAS-60 3xGFP11-FLAG-HA KI HEK 293T cells were generated using CRISPR-Cas9. Guide RNAs (gRNAs) were designed with the guide design tool from the Zhang lab (crispr.mit.edu) to target the RBM10 genomic region gRNA1, 5'- TGTCGGCCAGGATTCCTACG-3'; gRNA2, 5'- CCCGATAGTCGCCGTCTCGG-3'. Double-stranded DNA oligonucleotides corresponding to the gRNAs were inserted into pSpCas9(BB)-2A-GFP vector (Addgene, as a gift from F. Zhang, MIT, Cambridge, MA). A donor plasmid containing $300 \mathrm{bp}$ homology left-arm and $300 \mathrm{bp}$ homology right-arm sequence around the stop codon of MINAS-60, which are separated with 3xGFP11-FLAG$\mathrm{HA}$ tag and BamHI / Notl restriction sites was synthesized by GenScript, and a DNA sequence containing pGK promoter and hygromycin resistance gene were subcloned into the donor plasmid using the $\mathrm{BamH} 1$ and Notl restriction sites. An 
equal mixture of the gRNA and donor plasmids were transfected into HEK 293T cells using polyethyleneimine, and hygromycin selection was performed 2 days post-transfection. MINAS-60-3xGFP11-FLAG-HA KI cells were confirmed by genomic DNA PCR and sequencing.

Puromycin incorporation. SUnSET was used to measure protein synthesis ${ }^{63}$. Briefly, HEK 293T cells were grown to $80-90 \%$ confluency in 6 well plates, then growth media was replaced with media containing $1 \mu \mathrm{M}$ puromycin and cells were incubated at $37^{\circ} \mathrm{C}$ in a humidified atmosphere with $5 \% \mathrm{CO}_{2}$ for $1 \mathrm{~h}$ according to a previously published protocol ${ }^{56}$. The cells were then washed once with PBS, harvested and analyzed with western blotting.

Sucrose gradient profiling. HEK $293 \mathrm{~T}$ cells were seeded in $15 \mathrm{~cm}$ dishes at $2.5 \times 10^{7}$ cells per dish and cultured $24 \mathrm{~h}$. Cells were then treated with $100 \mu \mathrm{g} / \mathrm{mL}$ cycloheximide for $5 \mathrm{~min}$, washed with cold PBS containing $100 \mu \mathrm{g} / \mathrm{mL}$ cycloheximide twice, harvested and flash frozen until further processing.

For cytoplasmic polysome profiling, after thawing on ice, cells were lysed in polysome lysis buffer (20 mM HEPES-KOH pH 7.4, $100 \mathrm{mM} \mathrm{KCl}, 5 \mathrm{mM} \mathrm{MgCl}_{2}$, $100 \mu \mathrm{g} / \mathrm{mL}$ cycloheximide, $1 \mathrm{mM}$ DTT with $1 \%$ TritonX-100, Roche Complete protease inhibitor cocktail tablets and Ribonuclease Inhibitors (Promega N2511)), and incubated on ice for $10 \mathrm{~min}$, followed by centrifugation at $21,130 \mathrm{~g}, 4^{\circ} \mathrm{C}, 10$ min. The supernatants were normalized according to absorbance (A260) and

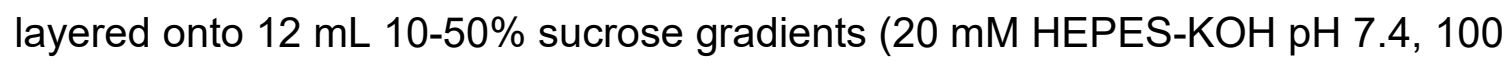
$\mathrm{mM} \mathrm{KCl}, 5 \mathrm{mM} \mathrm{MgCl} 2,100 \mu \mathrm{g} / \mathrm{mL}$ cycloheximide, $1 \mathrm{mM}$ DTT with Roche Complete protease inhibitor cocktail tablets and Ribonuclease Inhibitors), followed with centrifugation in an SW-41Ti rotor at $252,878 \mathrm{~g}, 4^{\circ} \mathrm{C}, 3 \mathrm{~h}$, then sampled using a Biocomp Gradient profiler (model 251) with constant monitoring of optical density at $254 \mathrm{~nm}$ using standard parameters. Data analysis was performed with Excel. 
839 For sucrose gradient sedimentation analysis of nuclear lysates, thawed cells 840 were suspended in $1 \mathrm{~mL}$ nuclear isolation buffer (10 mM HEPES-KOH pH 7.4, $841100 \mathrm{mM} \mathrm{KCl}, 5 \mathrm{mM} \mathrm{MgCl} 2$ with $0.5 \%$ NP40, Roche Complete protease inhibitor 842 cocktail tablets and Ribonuclease Inhibitors), and incubated on ice for $10 \mathrm{~min}$, 843 followed by centrifugation at $3,000 \mathrm{~g}, 4^{\circ} \mathrm{C}, 3 \mathrm{~min}$. The nuclear pellets were 844 suspended in $1 \mathrm{~mL}$ nuclear lysis buffer (20 mM HEPES-KOH pH 7.4, $300 \mathrm{mM}$ $845 \mathrm{KCl}, 5 \mathrm{mM} \mathrm{MgCl}$, $1 \mathrm{mM}$ DTT with 1\% TritonX-100, Roche Complete protease 846 inhibitor cocktail tablets and Ribonuclease Inhibitors), followed with sonication

847 (50\% intensity, 5 s pulse with 25 s rest, $5 \times$, MICROSON XL 2000), and 848 centrifugation at $21,130 \mathrm{~g}, 4^{\circ} \mathrm{C}, 10 \mathrm{~min}$. The supernatants were normalized by 849 A260 and layered onto $12 \mathrm{~mL} 10-50 \%$ sucrose gradients, centrifuged and 850 sampled as described above.

Crystal violet staining and cell cycle synchronization. $4 \times 10^{4} \mathrm{HEK} 293 \mathrm{~T}$ cells 853 were seeded in 12-well plates in triplicate, and fixed in 10\% formalin for $15 \mathrm{~min}$ at 854 RT every $24 \mathrm{~h}$. After washing with $\mathrm{ddH}_{2} \mathrm{O}$, cells were stained with $0.1 \%$ crystal 855 violet in methanol for $30 \mathrm{~min}$ at $\mathrm{RT}$ in the dark, followed with three $\mathrm{ddH}_{2} \mathrm{O}$ 856 washes and dried. The cells were then immersed in $1 \mathrm{~mL} 10 \%$ acetic acid with 857 shaking for $20 \mathrm{~min} .20 \mu \mathrm{L}$ of the solution was combined with $80 \mu \mathrm{L} \mathrm{ddH}_{2} \mathrm{O}$ in a 858 96-well plate, and the optical density at $590 \mathrm{~nm}$ was monitored with Synergy ${ }^{\mathrm{TM}}$ 859 HT.

861 For cell cycle synchronization, $1.5 \times 10^{5} \mathrm{HEK} 293 \mathrm{~T}$ cells were seeded in 12-well 862 plates and cultured overnight. Cells were then treated with $2 \mathrm{mM}$ thymidine for 16 $863 \mathrm{~h}$, followed with two PBS washes, and incubated with fresh media for $9 \mathrm{~h}$ before 864 the second $2 \mathrm{mM}$ thymidine block for $14 \mathrm{~h}$ following a previously published 865 protocol $^{64}$. Cells were washed with PBS, then incubated with fresh media to 866 release from the G1/S boundary, and fixed in $10 \%$ formalin for immunostaining or 867 harvested for western blotting.

\section{Northern blot}


870 Total RNA was extracted from HEK 293T cells using TRIzol reagent. To

871 determine changes in levels of LSU pre-rRNA intermediates, $3 \mu \mathrm{g}$ of total RNA

872 was run on a $1 \%$ agarose/ $1.25 \%$ formaldehyde gel in a $1.5 \mathrm{M}$ tricine/ $1.5 \mathrm{M}$

873 triethanolamine buffer, followed by an overnight transfer to a Hybond XL nylon

874 membrane (GE Healthcare, RPN 303S) in 10x saline-sodium citrate transfer

875 buffer after a brief $15 \mathrm{~min}$ soak in a $0.5 \mathrm{M}$ sodium hydroxide solution. Membranes

876 were then exposed to UV ( $254 \mathrm{~nm}$ ) to immobilize the RNA, followed by

877 incubation with denatured yeast tRNA for $1 \mathrm{~h}$ at $42^{\circ} \mathrm{C}$, and hybridized overnight at

$87837^{\circ} \mathrm{C}$ with $5^{\prime}$ end radiolabeled oligonucleotide probe (P4 5'-

879 CGGGAACTCGGCCCGAGCCGGCTCTCTCTTTCCCTCTCCG-3') in a solution

880 of $7.5 \times$ Denhardt's solution, $5 \times$ sodium chloride-sodium phosphate-EDTA buffer

881 with $0.1 \%$ SDS, as previously reported ${ }^{59}$. Membranes were also hybridized with a

882 7SL probe (7SL 5'-TGCTCCGTTTCCGACCTGGGCCGGTTCACCCCTCCTT-3')

883 as a loading control.

884

\section{Data availability}

886 Proteomic data were deposited under accession PXD026880. During review,

887 they can be accessed with username reviewer_pxd026880@ebi.ac.uk and

888 password 7dkNTlia.

889

890 Acknowledgements

891 We thank Franziska Bleichert and all members of the Slavoff and Baserga labs

892 for helpful conversations. This work was supported by a Searle Scholars

893 Program Award, an Odyssey Award from the Richard and Susan Smith Family

894 Foundation, and start-up funds from Yale University West Campus (to S. A. S.).

895 X.C. was supported in part by a Rudolph J. Anderson postdoctoral fellowship

896 from Yale University. A.K. was in part supported by an NIH Predoctoral Training

897 Grant (5T32GM06754 3-12). S.J.B, C.J.B. and C.M.H. were supported by R35

898 GM131687. C.M.H. was supported by an NSF GFRP.

899

900 Author contributions 

approved the final version of the manuscript.

\section{References}

1. Basrai, M.A., Hieter, P. \& Boeke, J.D. Small open reading frames: beautiful needles in the 
19. Sanghai, Z.A. et al. Modular assembly of the nucleolar pre-60S ribosomal subunit.

Nature 556, 126-129 (2018).

20. Micic, J. et al. Coupling of 5S RNP rotation with maturation of functional centers during large ribosomal subunit assembly. Nat Commun 11, 3751 (2020).

21. Pelletier, J., Thomas, G. \& Volarevic, S. Ribosome biogenesis in cancer: new players and therapeutic avenues. Nat Rev Cancer 18, 51-63 (2018).

22. Nyhus, C., Pihl, M., Hyttel, P. \& Hall, V.J. Evidence for nucleolar dysfunction in Alzheimer's disease. Rev Neurosci 30, 685-700 (2019).

23. Farley-Barnes, K.I., Ogawa, L.M. \& Baserga, S.J. Ribosomopathies: Old Concepts, New Controversies. Trends Genet 35, 754-767 (2019).

24. Mills, E.W. \& Green, R. Ribosomopathies: There's strength in numbers. Science 358(2017).

25. Hernandez, J. et al. Tumor suppressor properties of the splicing regulatory factor RBM10. RNA Biol 13, 466-72 (2016).

26. Inoue, A. et al. RBM10 regulates alternative splicing. FEBS Lett 588, 942-7 (2014).

27. Rodor, J., FitzPatrick, D.R., Eyras, E. \& Caceres, J.F. The RNA-binding landscape of RBM10 and its role in alternative splicing regulation in models of mouse early development. RNA Biol 14, 45-57 (2017).

28. Sutherland, L.C. et al. Splicing arrays reveal novel RBM10 targets, including SMN2 premRNA. BMC Mol Biol 18, 19 (2017).

29. Bechara, E.G., Sebestyen, E., Bernardis, I., Eyras, E. \& Valcarcel, J. RBM5, 6, and 10 differentially regulate NUMB alternative splicing to control cancer cell proliferation. $\mathrm{Mol}$ Cell 52, 720-33 (2013).

30. Dieterich, D.C., Link, A.J., Graumann, J., Tirrell, D.A. \& Schuman, E.M. Selective identification of newly synthesized proteins in mammalian cells using bioorthogonal noncanonical amino acid tagging (BONCAT). Proc Natl Acad Sci U S A 103, 9482-7 (2006).

31. Ma, J. et al. Improved Identification and Analysis of Small Open Reading Frame Encoded Polypeptides. Anal Chem 88, 3967-75 (2016).

32. Khitun, A. \& Slavoff, S.A. Proteomic Detection and Validation of Translated Small Open Reading Frames. Curr Protoc Chem Biol 11, e77 (2019).

33. Chu, Q. et al. Identification of Microprotein-Protein Interactions via APEX Tagging. Biochemistry 56, 3299-3306 (2017).

34. Wang, T., Liu, Y., Liu, Q., Cummins, S. \& Zhao, M. Integrative proteomic analysis reveals potential high-frequency alternative open reading frame-encoded peptides in human colorectal cancer. Life Sci 215, 182-189 (2018).

35. Kamiyama, D. et al. Versatile protein tagging in cells with split fluorescent protein. Nat Commun 7, 11046 (2016).

36. Iyer-Bierhoff, A. \& Grummt, I. Stop-and-Go: Dynamics of Nucleolar Transcription During the Cell Cycle. Epigenet Insights 12, 2516865719849090 (2019).

37. Andrews, J.O. et al. qSR: a quantitative super-resolution analysis tool reveals the cellcycle dependent organization of RNA Polymerase I in live human cells. Sci Rep 8, 7424 (2018).

38. Gorski, S.A., Snyder, S.K., John, S., Grummt, I. \& Misteli, T. Modulation of RNA polymerase assembly dynamics in transcriptional regulation. Mol Cell 30, 486-97 (2008).

39. Hernandez-Verdun, D. Assembly and disassembly of the nucleolus during the cell cycle. Nucleus 2, 189-94 (2011).

40. Cao, X. \& Slavoff, S.A. Non-AUG start codons: Expanding and regulating the small and alternative ORFeome. Exp Cell Res 391, 111973 (2020). 


\footnotetext{
993 41. Tan, L.Y. et al. Generation of functionally distinct isoforms of PTBP3 by alternative

994 splicing and translation initiation. Nucleic Acids Res 43, 5586-600 (2015).

995 42. Saghatelian, A. \& Couso, J.P. Discovery and characterization of smORF-encoded

996 bioactive polypeptides. Nat Chem Biol 11, 909-16 (2015).

997 43. Cox, J. et al. Accurate proteome-wide label-free quantification by delayed normalization

998

999

1000

1001

1002

1003

1004

1005

1006

1007

1008

1009

1010

1011

1012

1013

1014

1015

1016

1017

1018

1019

1020

1021

1022

1023

1024

1025

1026

1027

1028

1029

1030

1031

1032

1033

1034

1035

1036

1037

1038

1039

1040 and maximal peptide ratio extraction, termed MaxLFQ. Mol Cell Proteomics 13, 2513-26 (2014).

44. Liang, X. et al. Structural snapshots of human pre-60S ribosomal particles before and after nuclear export. Nat Commun 11, 3542 (2020).

45. Liu, Y., Deisenroth, C. \& Zhang, Y. RP-MDM2-p53 Pathway: Linking Ribosomal Biogenesis and Tumor Surveillance. Trends Cancer 2, 191-204 (2016).

46. Sondalle, S.B., Longerich, S., Ogawa, L.M., Sung, P. \& Baserga, S.J. Fanconi anemia protein FANCl functions in ribosome biogenesis. Proc Natl Acad Sci U S A 116, 2561-2570 (2019).

47. Bassler, J. \& Hurt, E. Eukaryotic Ribosome Assembly. Annu Rev Biochem 88, 281-306 (2019).

48. Wild, T. et al. A protein inventory of human ribosome biogenesis reveals an essential function of exportin 5 in 60S subunit export. PLOS Bio/ 8, e1000522 (2010).

49. Klemke, M., Kehlenbach, R.H. \& Huttner, W.B. Two overlapping reading frames in a single exon encode interacting proteins--a novel way of gene usage. EMBO J 20, 3849-60 (2001).

50. Inoue, A. RBM10: Structure, functions, and associated diseases. Gene 783, 145463 (2021).

51. Niceta, M. et al. TARP syndrome: Long-term survival, anatomic patterns of congenital heart defects, differential diagnosis and pathogenetic considerations. Eur J Med Genet 62, 103534 (2019).

52. Imielinski, M. et al. Mapping the hallmarks of lung adenocarcinoma with massively parallel sequencing. Cell 150, 1107-20 (2012).

53. Gagnon, M. et al. Potentiation of B2 receptor signaling by AltB2R, a newly identified alternative protein encoded in the human bradykinin B2 receptor gene. J Biol Chem 296, 100329 (2021).

54. Carvunis, A.R. et al. Proto-genes and de novo gene birth. Nature 487, 370-4 (2012).

55. Parker, M.D., Collins, J.C., Korona, B., Ghalei, H. \& Karbstein, K. A kinase-dependent checkpoint prevents escape of immature ribosomes into the translating pool. PLoS Biol 17, e3000329 (2019).

56. Farley-Barnes, K.I. et al. Diverse Regulators of Human Ribosome Biogenesis Discovered by Changes in Nucleolar Number. Cell Rep 22, 1923-1934 (2018).

57. Nguyen le, X.T., Raval, A., Garcia, J.S. \& Mitchell, B.S. Regulation of ribosomal gene expression in cancer. J Cell Physiol 230, 1181-8 (2015).

58. Xing, Y.H. et al. SLERT Regulates DDX21 Rings Associated with Pol I Transcription. Cell 169, 664-678 e16 (2017).

59. Ogawa, L.M. et al. Increased numbers of nucleoli in a genome-wide RNAi screen reveal proteins that link the cell cycle to RNA polymerase I transcription. Mol Biol Cell 32, 956973 (2021).

60. Cao, X. et al. Comparative Proteomic Profiling of Unannotated Microproteins and Alternative Proteins in Human Cell Lines. J Proteome Res 19, 3418-3426 (2020).

61. Gregory, B. et al. The small and large ribosomal subunits depend on each other for stability and accumulation. Life Sci Alliance 2(2019).
} 
1041 62. Weerapana, E., Speers, A.E. \& Cravatt, B.F. Tandem orthogonal proteolysis-activity-

63. Schmidt, E.K., Clavarino, G., Ceppi, M. \& Pierre, P. SUnSET, a nonradioactive method to 1045

1046

1047

64. Cao, X. et al. Histone H4K20 Demethylation by Two hHR23 Proteins. Cell Rep 30, 41524164 e6 (2020). 


\section{Supplementary Files}

This is a list of supplementary files associated with this preprint. Click to download.

- SupplementaryData1.xlsx

- Supplementaryinformation.pdf

- SupplementaryData2.xlsx

- SupplementaryData3.xlsx

- SupplementaryData5.xlsx

- SupplementaryData4.xlsx

- SupplementaryData6.xlsx 